\title{
Observer-Based Adaptive Iterative Learning Control for a Class of Nonlinear Time Delay Systems with Input Saturation
}

\author{
Jian-ming Wei, Yun-an Hu, and Mei-mei Sun \\ Department of Control Engineering, Naval Aeronautical and Astronautical University, Yantai 264001, China \\ Correspondence should be addressed to Yun-an Hu; hya507@sina.com
}

Received 19 May 2015; Revised 14 July 2015; Accepted 22 July 2015

Academic Editor: Xinkai Chen

Copyright (C) 2015 Jian-ming Wei et al. This is an open access article distributed under the Creative Commons Attribution License, which permits unrestricted use, distribution, and reproduction in any medium, provided the original work is properly cited.

\begin{abstract}
This paper presents an adaptive iterative learning control scheme for the output tracking of a class of nonlinear systems with unknown time-varying delays and input saturation nonlinearity. An observer is presented to estimate the states and linear matrix inequality (LMI) method is employed for observer design. The assumption of identical initial condition for ILC is relaxed by introducing boundary layer function. The possible singularity problem is avoided by introducing hyperbolic tangent function. The uncertainties with time-varying delays are compensated for by the combination of appropriate Lyapunov-Krasovskii functional and Young's inequality. Both time-varying and time-invariant radial basis function neural networks are employed to deal with system uncertainties. On the basis of a property of hyperbolic tangent function, the system output is proved to converge to a small neighborhood of the desired trajectory by constructing Lyapunov-like composite energy function in two cases, while keeping all the closed-loop signals bounded. Finally, a simulation example is presented to verify the effectiveness of the proposed approach.
\end{abstract}

\section{Introduction}

Over the past decades, tremendous research efforts have been made aiming at the development of systematic design methods for the iterative learning control (ILC) of nonlinear systems performing control task over a finite interval repeatedly. ILC has become the most suitable and effective control scheme for such repeatable control tasks because of its capacity of achieving perfect tracking by learning mechanism along iteration. Generally, according to the stability analysis tool, ILC can be classified into two categories: traditional ILC [1-4] and adaptive ILC (AILC) [5-10]. The basic principle of traditional ILC is to use information collected from previous execution to form the control action for current operation by a learning mechanism for purpose of improving performances from iteration to iteration. Furthermore, the stability conclusion of traditional ILC is usually obtained by using contraction mapping theorem and fixed point theorem. However, traditional ILC requires for the global Lipschitz continuous condition, which makes it difficult to apply it to certain nonlinear systems. Besides, traditional ILC uses contraction mapping theorem rather than Lyapunov method as the key principle of stability analysis, which makes it difficult to relax the global Lipschitz condition to local Lipschitz or even non-Lipschitz condition and cooperate with the mainstream methods of nonlinear control theory, such as adaptive control and neural control. To relax the constraints of traditional ILC and extend it to a broader range, some researchers tried to introduce the idea of adaptive control into ILC and proposed adaptive iterative learning control (AILC). AILC takes advantage of both adaptive control and ILC, which successfully overcomes the restriction of global Lipschitz condition; thus it enables us to use fuzzy logic systems or neural networks as approximators to deal with nonlinear uncertainties. In general, the control parameters of AILC methods are tuned along the iteration axis, and the so-called composite energy function (CEF) [5] is usually constructed to analyze the stability and convergence property of the closed-loop systems. The past decade has witnessed great progress in AILC of uncertain nonlinear systems [6-10].

In practice, control of systems with time delays has always been a meaning research, since time delay can be often encountered in a wide range of physical systems and devices, such as turbojet engines, aircraft systems, microwave oscillators, nuclear reactors, and chemical processes [11, 12]. The existence of time delays in a system may degrade the 
control performance and even at worst may become a source of instability. Thus, the investigation of time delay in systems has always been an active topic for control engineers. Consequently, stabilization problem of control systems with time delay has received much attention for several decades and a large number of research results have been reported in the literature that deal with various analysis and design problems [11-16]. However, in the field of AILC, only a few results are available for nonlinear systems with time delays [17-19]. In [17], an AILC strategy was developed for a class of scalar systems with unknown time-varying delay and then extended to a class of high-order systems with both time-varying and time-invariant parameters, where the unknown time-varying parameter was estimated in the iterative learning process. However, the proposed controller in [17] requires that the uncertainties in the system satisfy local Lipschitz condition and nonlinear parameterized condition such that adaptive learning laws can be used to estimate the unknown timevarying parameters. In $[18,19]$, we designed an AILC scheme for a class of nonlinearly parameterized systems and an RBF NN-based AILC for class of unparameterized systems, respectively, where the systems in two papers are with both unknown time-varying delays and unknown dead zone input. However, all of the aforementioned results are on systems with time delay states. As for systems with time delay outputs, to the best of our knowledge, there are no works reported in the literature.

Other than time delay, another challenging problem in control of nonlinear systems lies in the existence of nonsmooth and nonlinear characteristics such as dead zone, hysteresis, saturation, and backlash. Among them, the significance of controller design for systems with saturation can be overemphasized, as any control systems depending on actuators have physical limitations, for example, mechanical actuators and aircraft. The existence of saturation can severely limit system performances and usually leads to undesirable inaccuracies and even instability [20]. Therefore, the control design for nonlinear systems preceded by input saturation is a challenging but worthwhile and necessary issue. For control systems with input saturation, many results have been published in the past several decades [20-26]. To address such a problem, the principal work is to model the input saturation nonlinearity for controller design. Generally, saturation is modeled as $u=\operatorname{sat}(v)=v+\Delta u$, where $v$ and $u$ are the input and output of saturation nonlinearity, respectively, and $\Delta u$ denotes the difference between the actual control effect $u$ and input $v$. In [20], the hyperbolic tangent function is employed in the model of input saturation. Like control design for systems with time delays, only a few results are available at present stage from the viewpoint of ILC. In [7], an iterative learning control scheme is designed for a class of nonlinear uncertain systems with input saturation and the analysis of convergence in the iteration domain was presented on the basis of composite energy function consisting of both input and output information. In [27] an adaptive iterative learning control strategy for high-speed trains with unknown speed delays and control input saturation is designed to address speed trajectory tracking problem. It turns out that control design in the presence of both input saturation and time delays is even more difficult. As far as we know, there are few works reported from the viewpoint of AILC to deal with nonlinear systems with saturation nonlinearity and time delays in the literature at present stage.

In the control community, state feedback control is very powerful for nonlinear systems as the full information of the state vectors is assumed to be accessible for feedback. However, in many real world control systems, only the measured output information, rather than the full state information, is available for feedback. For systems with only output measurable, observer-based output feedback controller has been proven to be the most effective scheme, which is a dynamic output feedback controller that estimates the system states online. Over the past decades, there has been a considerable development in various observer design methodologies using different approaches [28-35]. Although so many results have been developed, only a few results are available from the point of AILC [36-42]. How to design an AILC for nonlinear systems using only output measurement is an interesting and challenging issue. Tayebi and $\mathrm{Xu}$ proposed an observer-based iterative learning control scheme for the tracking problem of a class of time-varying nonlinear systems and gave the sufficient conditions for the boundedness and the convergence to zero of the estimation error by using contraction mapping method [36]. In [37], a learning control approach combined with an observer was developed for output tracking of a class of nonlinear systems with time-varying parametric uncertainties and the Lyapunov-like energy function was employed to facilitate convergence analysis. Wang and Chien introduced an error observer to design an iterative learning controller for robotic systems, where a robust learning component using a filtered fuzzy neural network was presented to solve the problem of unknown nonlinearities [38]. Subsequently, the results in [38] were extended to SISO nonlinear system [39], MIMO nonlinear systems [40], and MIMO nonlinear systems with delayed output [41]. Chen et al. extended the result in [36] and proposed an observer-based AILC for nonlinear systems with unknown time-varying parametric uncertainties and the delayed output, where the Lyapunov-Krasovskii-like composite energy function was constructed to prove the boundedness of all closed-loop signals and the convergence of output tracking error [42]. But unfortunately the system nonlinearities must satisfy global Lipschitz continuous condition and the observer design depends on the known system's nonlinear functions. Even though the AILC problem for nonlinear systems using only output measurement has been investigated in the above works, it is noted that the dynamics of nonlinear systems in these works need to satisfy some special structures or conditions.

Motivated by the above observations, in this paper we consider the observer-based AILC problem for a class of nonlinear systems with unknown time-varying delays preceded by input saturation. To the best of our knowledge, up till now no works have been reported in the field of AILC to deal with such kinds of systems. In the proposed AILC scheme, both time-varying and time-invariant radial basis function neural network are utilized to form the iterative learning controller and a CEF along both time and iteration axes is constructed 
to analyze the stability and convergence property. Theoretical analysis and simulation example show that the proposed approach can guarantee that all the signals are bounded and tracking errors converge to a small neighbourhood of the origin. The main contributions of the proposed AILC scheme are highlighted as follows. (1) As far as we know, it is the first time, in the literature, that observer-based AILC problem of unknown nonlinear systems with unknown time-varying time delays and input saturation is investigated. (2) Both time-varying and time-invariant RBF neural networks are used to approximate the unknown time-varying functions and uncertainty that is independent of time variable. (3) Hyperbolic tangent function is employed to handle the singularity problem encountered in Lyapunov synthesis. (4) The boundary layer function is introduced to remove the requirement for identical initial condition which is required for the majority of ILC schemes.

The organization of this paper is as follows. The problem formulation and preliminaries are given in Section 2. In Section 3, the observer and AILC scheme are developed. Results of extensive simulation studies are presented to demonstrate the validity of the proposed scheme in Section 4, followed by conclusions in Section 5 .

Throughout this paper, the following notations will be used. (1) $\sigma$ denotes the integral variable. (2) $|\cdot|$ denotes the absolute value of a scalar function. (3) $\|\cdot\|$ denotes the Euclidean norm or any other consistent norm of a vector or matrix. (4) $N$ is the set of nonnegative integers. (5) For a signal vector $r_{k}(t)$, define $\left\|r_{k}(t)\right\|_{L_{T}^{\infty}}=\max _{(k, t) \in N \times[0, T]}\left\|r_{k}(t)\right\|$ and $\left\|r_{k}(t)\right\|_{L_{T}^{2}}=\int_{0}^{T}\left\|r_{k}(\sigma)\right\|^{2} \mathrm{~d} \sigma$. If $\left\|r_{k}(t)\right\|_{L_{T}^{\infty}}<\infty$, we say that $r_{k}(t)$ is bounded in $L_{T}^{\infty}$-norm, which is denoted by $r_{k}(t) \in L_{T}^{\infty}$. Similarly, we denote the boundedness of $r_{k}(t)$ in $L_{T}^{2}$-norm by $r_{k}(t) \in L_{T}^{2}$. Obviously, the boundedness in $L_{T}^{\infty}$-norm implies the boundedness in $L_{T}^{2}$-norm because $\left\|r_{k}(t)\right\|_{L_{T}^{2}} \leq$ $T\left\|r_{k}(t)\right\|_{L_{T}^{\infty}}^{2}$.

\section{Problem Formulation and Preliminaries}

2.1. Problem Formulation. In this paper, we consider a class of nonlinear time delay systems with input saturation which runs on a finite time interval $[0, T]$ repeatedly

$$
\begin{aligned}
\dot{x}_{i, k}(t)= & x_{i+1, k}(t), \quad i=1, \ldots, n-1, \\
\dot{x}_{n, k}(t)= & f\left(x_{k}(t), t\right)+h\left(y_{\tau, k}(t), t\right)+u_{k}\left(v_{k}(t)\right) \\
& +d(t), \\
y_{k}(t)= & x_{1, k}(t), \quad t \in[0, T], \\
y_{k}(t)= & \omega(t), \quad t \in\left[-\tau_{\max }, 0\right),
\end{aligned}
$$

where $k \in N$ denotes the index of iteration; $y_{k}(t) \in R$ and $x_{i, k}(t) \in R, i=1, \ldots, n$, are the system output and states, respectively; $x_{k}(t) \triangleq\left[x_{1, k}(t), \ldots, x_{n, k}(t)\right]^{T}$ are the delayfree state vectors; $\tau_{i}(t)$ are unknown time-varying delays and $y_{\tau_{i}, k} \triangleq y_{k}\left(t-\tau_{i}(t)\right), i=2, \ldots, n$, and $y_{\tau, k}(t)=$ $\left[y_{\tau_{1}, k}(t), \ldots, y_{\tau_{n}, k}(t)\right]^{T} ; f(\cdot, \cdot)$ and $g(\cdot, \cdot)$ are unknown smooth functions; $h(\cdot, \cdot)$ is an unknown smooth function of time delay output with upper bound. $d(t)$ is unknown bounded external disturbance. $u_{k}\left(v_{k}(t)\right)$ denotes the saturation nonlinearity. $\omega(t)$ denotes the initial function for delayed output. The states are assumed to be unavailable for measurement and only output is measurable. The system is bounded-inputbounded-output (BIBO) stable. In the subsequent parts, the variable $t$ will be omitted when no confusions arise.

Our design objective is to determine an adaptive iterative controller for system (1) to steer the output $y_{k}$ following a desired reference signal $y_{d}$ over $[0, T]$ as $k \rightarrow \infty$, while ensuring that all the signals in the closed-loop system remain bounded.

Define the desired trajectory as $x_{d}=\left[y_{d}, \dot{y}_{d}, \ldots, y_{d}^{(n-1)}\right]^{T}$, which is a vector of $y_{d}$ up to its $(n-1)$ th time derivative. To facilitate control system design, the following reasonable assumptions are made.

Assumption 1. The unknown time-varying delays $\tau_{i}(t)$ satisfy $0 \leq \tau_{i}(t) \leq \tau_{\max }, \dot{\tau}_{i}(t) \leq \kappa<1, i=1,2, \ldots, n$, where $\tau_{\max }$ is known and $\kappa$ is an unknown positive constant.

Assumption 2. The unknown smooth function $h(\cdot, \cdot)$ satisfies the following inequality:

$$
\left|h\left(y_{\tau, k}, t\right)\right| \leq \sum_{j=1}^{n} \rho_{j}\left(y_{\tau_{j}, k}(t)\right)
$$

where $\rho_{j}(\cdot)$ are unknown positive smooth functions.

Assumption 3. The desired reference signal $y_{d}(t)$ up to its $n$th derivative is continuous, bounded, and available.

Assumption 4. The unknown external disturbance $d(t)$ is bounded; that is, $|d(t)| \leq D_{1}$, with $D_{1}$ as an unknown constant.

Assumption 5. The initial state vector is chosen as $\omega(t)=$ $y_{d}(t), t \in\left[-\tau_{\max }, 0\right)$.

Remark 6. Assumption 1 is usually necessary in the control problem of systems with unknown time-varying delays, which ensures that the time delay terms can be compensated for by using Lyapunov-Krasovskii functional. And this assumption is more relaxed than that in [17] which requires for a priori knowledge of $\kappa$.

Remark 7. Compared with the assumption of time-varying parameterization with known upper bound functions in [17], Assumption 2 is much more relaxed and can be easily satisfied.

Remark 8. Assumption 5 is only for analytical purpose and of no practical meaning.

2.2. Input Saturation. In this paper, the output of a control $u_{k}$ with input $v_{k}$ subjected to the condition of saturation is given by 


$$
u_{k}= \begin{cases}v_{k}, & \left|v_{k}\right|<u_{M} \\ u_{M} \operatorname{sgn}\left(v_{k}\right), & \left|v_{k}\right| \geq u_{M},\end{cases}
$$

where $u_{M}$ is the bound of input $v_{k}$. For convenience of design, we rewrite the saturation nonlinearity as

$$
u_{k}=\beta\left(v_{k}\right)+d_{1}\left(v_{k}\right)
$$

with $\beta\left(v_{k}\right)=u_{M} \times \tanh \left(v_{k} / u_{M}\right)$. The part of the control effect that cannot be implemented is defined as

$$
d_{1}\left(v_{k}\right)=u_{k}-\beta\left(v_{k}\right) .
$$

Obviously, $d_{1}\left(v_{k}\right)$ is bounded by

$$
\begin{aligned}
\left|d_{1}\left(v_{k}\right)\right| & =\left|\operatorname{sat}\left(v_{k}\right)-\beta\left(v_{k}\right)\right| \leq u_{M}(1-\tanh (1)) \\
& =D_{2} .
\end{aligned}
$$

A graphic presentation of saturation model is shown in Figure 1.

2.3. RBF Neural Networks. In control engineering, two types of artificial neural networks are usually used to approximate unknown smooth functions, which specifically are linearly parameterized neural networks (LPNNs) and multilayer neural networks (MNNs). As a kind of LPNNs, the radial basis function (RBF) neural network (NN) [43] is usually used as a tool to model unknown nonlinear functions owing to its nice approximation capabilities. The RBF NN can be seen as a two-layer network in which hidden layer performs a fixed nonlinear transformation with no adjustable parameters; that is, the input space is mapped into a new space. The output layer then combines the outputs in the latter space linearly. Generally, the RBF NN approximates the continuous function $Q(Z): R^{q} \rightarrow R$ as follows:

$$
Q_{\mathrm{nn}}(Z)=W^{T} \phi(Z)
$$

where $Z \in \Omega_{Z} \subset R^{q}$ is the input vector, $W=\left[w_{1}, w_{2}, \ldots\right.$, $\left.w_{l}\right]^{T} \in R^{l}$ is the weight vector, and the NN node number $l>1$; and $\phi(Z)=\left[\varphi_{1}(Z), \ldots, \varphi_{l}(Z)\right]^{T}$, with $\varphi_{i}(Z)$ as the commonly used Gaussian functions; that is, $\varphi_{i}(Z)=$ $e^{-\left(Z-\mu_{i}\right)^{T}\left(Z-\mu_{i}\right) / \sigma_{i}^{2}}, i=1, \ldots, l$, where $\mu_{i}=\left[\mu_{i 1}, \mu_{i 2}, \ldots, \mu_{i q}\right]$ is the center of the receptive field and $\sigma_{i}$ is the width of the Gaussian function. It has been proven that if $l$ is chosen sufficiently large, $W^{T} \phi(Z)$ can approximate any continuous function, $Q(Z)$, over a compact set $\Omega_{Z} \subset R^{q}$ to any arbitrary accuracy in the form of $Q(Z)=W^{* T} \phi(Z)+\varepsilon(Z)$, for all $Z \in \Omega_{Z} \subset R^{q}$ where $W^{*}$ is the ideal constant weight vector and $\varepsilon(Z)$ is the approximation error which is bounded over the compact set; that is, $|\varepsilon(Z)| \leq \varepsilon^{*}$, for all $Z \in \Omega_{Z}$, where $\varepsilon^{*}>0$ is an unknown constant. The ideal weight vector $W^{*}$ is an "artificial" quantity required for analytical purposes. $W^{*}$ is defined as the value of $W$ that minimizes $|\varepsilon(Z)|$ for all $Z \in \Omega_{Z} \subset R^{q}$; that is, $W^{*}:=\operatorname{argmin}_{W \in R^{l}}\left\{\sup _{Z \in \Omega_{Z}} \mid Q(Z)-\right.$ $\left.W^{T} \phi(Z) \mid\right\}$.

When neural networks are used to approximate unknown functions, updating laws for estimating the weight vector

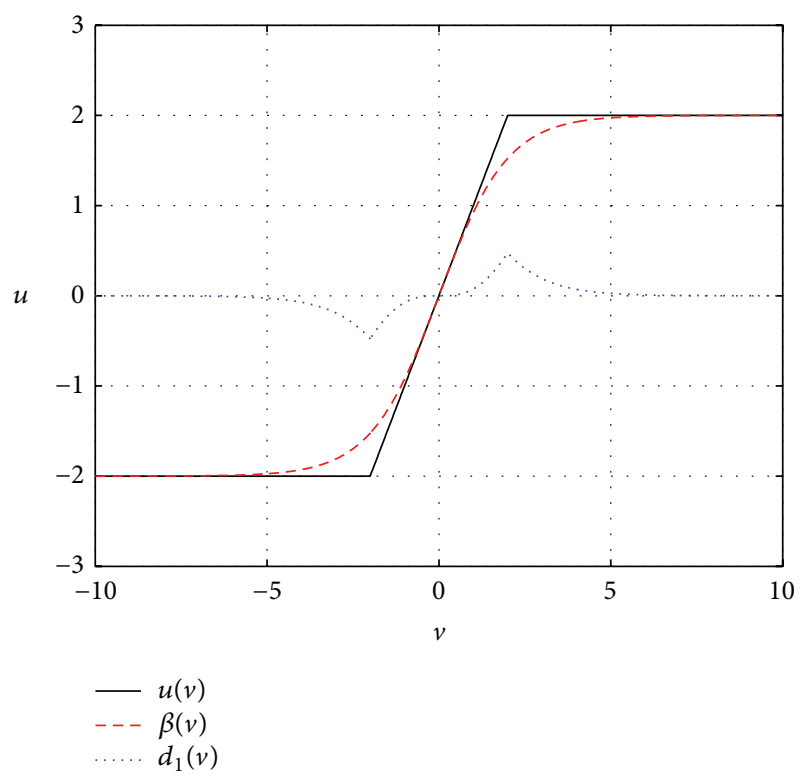

FIGURE 1: Saturation model.

need to be designed. In the early stage, the gradient-based back-propagation algorithms and their variants are the most popular algorithms for training neural networks. Along with the applications in traditional adaptive control framework, differential type updating laws were developed in the control scheme design and stability analysis by using Lyapunov method. Over the past few decades, large numbers of adaptive neural control schemes have been presented [11-13, 21, 24, 26]. However, difficulties arise when using (7) to approximate unknown time-varying function $Q(Z, t)$, especially when $Q(Z, t)$ is fast time-varying. In order to deal with this problem, a kind of time-varying neural networks is proposed to approximate the unknown time-varying functions in the form of $Q(Z, t)=W^{* T}(t) \phi(Z)+\varepsilon(Z, t)$, whose ideal weight vector $W^{*}(t)$ is time-varying [44, 45]. Difference type learning law is designed and the named CEF method is exploited to obtain the convergence and stability results.

In this paper, we will employ both time-varying $\mathrm{RBF}$ neural networks proposed in $[44,45]$ and time-invariant RBF NNs (7) as the approximator to overcome the design problem from the uncertainties.

2.4. Schur Complement Lemma [46]. In this paper, the following lemma is used.

Lemma 9. The LMI

$$
S=\left[\begin{array}{ll}
S_{11} & S_{12} \\
S_{21} & S_{22}
\end{array}\right]<0,
$$

where $S_{11}=S_{11}^{T}$ and $S_{22}=S_{22}^{T}$, is equivalent to

$$
\begin{aligned}
S_{22} & <0, \\
S_{11}-S_{12} S_{22}^{-1} S_{12}^{T} & <0 .
\end{aligned}
$$




\section{Observer and Adaptive Neural Iterative Learning Controller Design}

3.1. Observer Design. Rewrite system (1) as

$$
\begin{aligned}
\dot{x}_{k} & =A x_{k}+K_{0} y_{k}+B\left[f\left(x_{k}, t\right)+h\left(y_{\tau, k}, t\right)+\beta\left(v_{k}\right)\right. \\
& \left.+d_{1}\left(v_{k}\right)+d(t)\right]
\end{aligned}
$$

with

$$
\begin{aligned}
& K_{o}=\left[k_{1}, k_{2}, \ldots, k_{n}\right]^{T} \text {, } \\
& A=\left[\begin{array}{cccc}
-k_{1} & & \\
-k_{2} & & I_{n-1} & \\
\vdots & & & \\
-k_{n} & 0 & \cdots & 0
\end{array}\right] \text {, } \\
& B=[0, \ldots, 0,1]^{T} \text {, }
\end{aligned}
$$

where $I_{n-1}$ is a unit square matrix with $n-1$ dimensions. $K_{0} \in R^{n}$ can be selected such that $A$ is a strict Hurwitz matrix. Thus, given a matrix $Q>0$, there exists $P>0$ satisfying the following inequality:

$$
A^{T} P+P A+\frac{n+3}{\lambda} P P^{T}<-Q
$$

where $\lambda$ is a positive scalar.

Remark 10. In this paper, to solve inequality (12), we decompose $A=\bar{A}+K_{0} \bar{B}$ where

$$
\begin{aligned}
& \bar{A}=\left[\begin{array}{lll}
0 & & \\
\vdots & I_{n-1} & \\
0 & \cdots & 0
\end{array}\right], \\
& \bar{B}=[-1,0, \ldots, 0] .
\end{aligned}
$$
LMI

Based on Lemma 9, (12) is equivalent to the following

$$
\left[\begin{array}{cc}
P \bar{A}+M \bar{B}+\bar{B}^{T} M^{T}+\bar{A}^{T} P+Q & P \\
P & -\frac{\lambda}{n+3} I_{n}
\end{array}\right]<0,
$$

where $Q$ is a given positive definite matrix and $I_{n}$ is a unit matrix. Furthermore, it should be noted that $P, M$, and $\lambda$ can be computed simultaneously via MATLAB LMI toolbox, and the observer gain matrix is obtained by $K_{0}=P^{-1} M$.

For simplicity, define $\bar{d}(t)=d_{1}\left(v_{k}\right)+d(t)$. Then it is obvious that $|\bar{d}(t)| \leq D_{0}$ with $D_{0}=D_{1}+D_{2}$. To estimate the states of system (10), design the observer as

$$
\begin{aligned}
& \dot{\hat{x}}_{k}=A \widehat{x}_{k}+K_{o} y_{k}+B\left[\Psi_{k}+v_{k}\right], \\
& \widehat{y}_{k}=\widehat{x}_{1, k},
\end{aligned}
$$

where $\Psi_{k}$ will be given later. For subsequent design, we define $\Delta v_{k}=\beta\left(v_{k}\right)-v_{k}$ which describes the effect of input saturation and can be effectively approximated by using a dynamic neural network.

Define $z_{k} \triangleq\left[z_{1, k}, z_{2, k}, \ldots, z_{n, k}\right]=x_{k}-\widehat{x}_{k}$; then we can obtain the dynamic of observer error by subtracting (15) from (10):

$$
\dot{z}_{k}=A z_{k}+B\left[F\left(x_{k}, t\right)+h\left(y_{\tau, k}, t\right)+\bar{d}(t)-\Psi_{k}\right],
$$

where $F\left(x_{k}, t\right)=f\left(x_{k}, t\right)+\Delta v_{k}$. Choose a positive function of observer error as $V_{z_{k}}=z_{k}^{T} P z_{k}$; taking the time derivative of $V_{z_{k}}$ yields

$$
\begin{aligned}
\dot{V}_{z_{k}}= & z_{k}^{T}\left(A^{T} P+P A\right) z_{k} \\
& +2 z_{k}^{T} P B\left[F\left(x_{k}, t\right)+h\left(y_{\tau, k}, t\right)+\bar{d}(t)-\Psi_{k}\right] .
\end{aligned}
$$

Considering Assumption 2 and utilizing Young's inequality, we can have

$$
\begin{aligned}
2 z_{k}^{T} P B h\left(y_{\tau, k}, t\right) & \leq 2\left|z_{k}^{T} P B\right|\left|h\left(y_{\tau, k}, t\right)\right| \\
& \leq \frac{n}{\lambda} z_{k}^{T} P P^{T} z_{k}+\lambda \sum_{j=1}^{n} \rho_{j}^{2}\left(y_{\tau_{j}, k}(t)\right), \\
2 z_{k}^{T} P B \bar{d}(t) & \leq \frac{1}{\lambda} z_{k}^{T} P P^{T} z_{k}+\lambda D_{0}^{2} .
\end{aligned}
$$

To compensate for the time delay term, consider the following Lyapunov-Krasovskii functional:

$$
V_{U_{k}}(t)=\frac{\lambda}{(1-\kappa)} \sum_{j=1}^{n} \int_{t-\tau_{j}(t)}^{t} \rho_{j}^{2}\left(y_{k}(\sigma)\right) \mathrm{d} \sigma .
$$

Recalling Assumption 1, differentiating (20) with respect to time leads to

$$
\begin{aligned}
\dot{V}_{U_{k}}(t) & =\frac{\lambda}{(1-\kappa)} \sum_{j=1}^{n} \rho_{j}^{2}\left(y_{k}\right)-\lambda \sum_{j=1}^{n} \frac{1-\dot{\tau}_{j}(t)}{(1-\kappa)} \rho_{j}^{2}\left(y_{\tau_{j}, k}\right) \\
& \leq \frac{\lambda}{(1-\kappa)} \sum_{j=1}^{n} \rho_{j}^{2}\left(y_{k}\right)-\lambda \sum_{j=1}^{n} \rho_{j}^{2}\left(y_{\tau_{j}, k}\right) .
\end{aligned}
$$

Combining (17)-(19) and (21), it follows that

$$
\begin{aligned}
\dot{V}_{z_{k}}+\dot{V}_{U_{k}} \leq & z_{k}^{T}\left(A^{T} P+P A+\frac{n+1}{\lambda}\right) z_{k} \\
& +2 z_{k}^{T} P B\left[F\left(x_{k}, t\right)-\Psi_{k}\right] \\
& +\frac{\lambda}{(1-\kappa)} \sum_{j=1}^{n} \rho_{j}^{2}\left(y_{k}\right)+\lambda D_{0}^{2} .
\end{aligned}
$$

To overcome the difficulty from unknown time-varying function $F\left(x_{k}, t\right)$, we utilize time-varying RBF NN to approximate $F\left(x_{k}, t\right)$ on the compact set $\Omega=\left\{x_{k}\right\} \subset R^{n}$ which is specified by

$$
F\left(x_{k}, t\right)=W^{* T}(t) \phi\left(x_{k}\right)+\varepsilon\left(x_{k}, t\right),
$$


where the Gaussian basis function is given by $\phi\left(x_{k}\right)=$ $\left[\varphi_{i}\left(x_{k}\right), \varphi_{2}\left(x_{k}\right), \ldots, \varphi_{l}\left(x_{k}\right)\right]^{T} \in R^{l}$ with $\varphi_{i}\left(x_{k}\right)=\exp \left(-\| x_{k}-\right.$ $\left.\mu_{i} \|^{2} / \sigma_{i}^{2}\right), \mu_{i} \in R^{n}$ and $\sigma_{i} \in R$, denoting the centers and widths, respectively. $W^{*}(t)$ and $\varepsilon\left(x_{k}, t\right)$ are bounded by $\sup _{t \in[0, T]}\left\{\left\|W^{*}(t)\right\|\right\} \leq \varepsilon_{W}$ and $\sup _{t \in[0, T]}\left\{\left|\varepsilon\left(x_{k}, t\right)\right|\right\} \leq \varepsilon_{0}$. Consequently, we can determine that

$$
\Psi_{k}=\widehat{W}_{k}^{T}(t) \phi\left(\widehat{x}_{k}\right) .
$$

Then we can have

$$
\begin{aligned}
& 2 z_{k}^{T} P B\left[F\left(x_{k}, t\right)-\Psi_{k}\right]=2 z_{k}^{T} P B\left[W^{* T}(t) \phi\left(x_{k}\right)\right. \\
& \left.\quad+\varepsilon\left(x_{k}, t\right)-\widehat{W}_{k}^{T} \phi\left(\widehat{x}_{k}\right)\right]=2 z_{k}^{T} P B\left[W^{* T}(t) \phi\left(x_{k}\right)\right. \\
& -W^{* T}(t) \phi\left(\widehat{x}_{k}\right)+\varepsilon\left(x_{k}, t\right)+W^{* T}(t) \phi\left(\widehat{x}_{k}\right) \\
& \left.-\widehat{W}_{k}^{T} \phi\left(\widehat{x}_{k}\right)\right]=2 z_{k}^{T} P B\left[W^{* T}(t) \widetilde{\phi}\left(x_{k}, \widehat{x}_{k}\right)\right. \\
& \left.\quad+\varepsilon\left(x_{k}, t\right)-\widetilde{W}_{k}^{T} \phi\left(\widehat{x}_{k}\right)\right],
\end{aligned}
$$

where $\widetilde{W}_{k}=\widehat{W}_{k}-W^{*}$ denotes the estimation error, $\widetilde{\phi}\left(x_{k}, \widehat{x}_{k}\right)=\phi\left(x_{k}\right)-\phi\left(\widehat{x}_{k}\right) \cdot \widetilde{\phi}\left(x_{k}, \widehat{x}_{k}\right)$ is bounded by $\widetilde{\phi}^{T}\left(x_{k}, \widehat{x}_{k}\right) \widetilde{\phi}\left(x_{k}, \widehat{x}_{k}\right) \leq 4 l$. Then by using Young's inequality it is clear that

$$
\begin{gathered}
2 z_{k}^{T} P B\left[W^{* T}(t) \tilde{\phi}\left(x_{k}, \widehat{x}_{k}\right)+\varepsilon\left(x_{k}\right)\right] \\
\leq \frac{2}{\lambda} z_{k}^{T} P P^{T} z_{k}+4 \lambda l \varepsilon_{W}^{2}+\lambda \varepsilon_{0}^{2} .
\end{gathered}
$$

Substituting (25) and (26) back into (22) and applying (12) we have

$$
\begin{aligned}
\dot{V}_{z_{k}}+\dot{V}_{U_{k}} \leq & z_{k}^{T}\left(A^{T} P+P A+\frac{n+3}{\lambda} P P^{T}\right) z_{k} \\
& -2 z_{k}^{T} P B \widetilde{W}_{k}^{T} \phi\left(\widehat{x}_{k}\right)+\frac{\lambda}{(1-\kappa)} \sum_{j=1}^{n} \rho_{j}^{2}\left(y_{k}\right) \\
& +\lambda D_{0}^{2}+4 \lambda l \varepsilon_{W}^{2}+\lambda \varepsilon_{0}^{2} \\
\leq & -z_{k}^{T} Q z_{k}-2 z_{k}^{T} P B \widetilde{W}_{k}^{T} \phi\left(\widehat{x}_{k}\right) \\
& +\frac{\lambda}{(1-\kappa)} \sum_{j=1}^{n} \rho_{j}^{2}\left(y_{k}\right)+\lambda D_{0}^{2}+4 \lambda \varepsilon_{W}^{2} \\
& +\lambda \varepsilon_{0}^{2} \\
\leq & -\lambda{ }_{\min }(Q)\left\|z_{k}\right\|^{2}-2 z_{k}^{T} P B \widetilde{W}_{k}^{T} \phi\left(\widehat{x}_{k}\right) \\
& +\frac{\lambda}{(1-\kappa)} \sum_{j=1}^{n} \rho_{j}^{2}\left(y_{k}\right)+\lambda D_{0}^{2}+4 \lambda l \varepsilon_{W}^{2} \\
& +\lambda \varepsilon_{0}^{2} .
\end{aligned}
$$

3.2. Adaptive Neural Iterative Learning Controller Design. Define errors $e_{1, k}=\widehat{x}_{1, k}-y_{d}, e_{i, k}=\widehat{x}_{i, k}-y_{d}^{(i-1)}, i=2, \ldots, n-1$, and $e_{n, k}=\widehat{x}_{n, k}-y_{d}^{(n-1)}$. Then we make the assumptions on initial errors.
Assumption 11. Identical initial condition is satisfied for original system (1) and observer (15); that is, $z_{i, k}(0)=0$, $i=1,2, \ldots, n$.

Assumption 12. Identical initial condition is not necessary for $e_{i, k}$; that is, the initial state errors $e_{i, k}(0)$ at each iteration are not necessarily zero small and fixed but are assumed to be bounded.

Define the filtered tracking error as $e_{s k}=\left[\begin{array}{ll}\Lambda^{T} & 1\end{array}\right] e_{k}$, where $\Lambda=\left[\lambda_{1}, \lambda_{2}, \ldots, \lambda_{n-1}\right]^{T}$ and $\lambda_{1}, \ldots, \lambda_{n-1}$ are chosen such that the polynomial $H(s)=s^{n-1}+\lambda_{n-1} s^{n-2}+\cdots+\lambda_{1}$ is a Hurwitz polynomial. It is obvious that if $e_{s k}$ approaches zero as $k \rightarrow \infty$, then $\left\|e_{k}\right\|$ will converge to the origin asymptotically.

Based on Assumption 12, there exist known constants $\varepsilon_{i}$, such that $\left|e_{i, k}(0)\right| \leq \varepsilon_{i}, i=1,2, \ldots, n$, for any $k \in N$. In order to overcome the uncertainty from initial tracking error, we define an auxiliary function $[9,10]$ as

$$
\begin{aligned}
s_{k} & =e_{s k}-\eta(t) \operatorname{sat}\left(\frac{e_{s k}}{\eta(t)}\right), \\
\eta(t) & =\varepsilon e^{-K t},
\end{aligned}
$$

where $\varepsilon=\left[\begin{array}{ll}\Lambda^{T} & 1\end{array}\right]\left[\varepsilon_{1}, \varepsilon_{2}, \ldots, \varepsilon_{n}\right]^{T}, K>0$. The saturation function sat $(\cdot)$ is defined as

$$
\operatorname{sat}\left(\frac{e_{s k}}{\eta(t)}\right)= \begin{cases}1, & \text { if } e_{s k}>\eta(t), \\ \frac{e_{s k}}{\eta(t)}, & \text { if }\left|e_{s k}\right| \leq \eta(t), \\ -1, & \text { if } e_{s k}<-\eta(t) .\end{cases}
$$

Remark 13. $\eta(t)$ is called time-varying boundary layer function. It is obvious that $\eta(t)$ decreases along time axis, which satisfies initial condition $\eta(0)=\varepsilon$ and $0<\eta(T) \leq \eta(t)$, for all $t \in[0, T]$. If $s_{k}(t)$ can converge to zero for all $t \in[0, T]$ as $k \rightarrow \infty$, then $e_{s k}$ will be bounded by $\eta(t)$.

According to initial condition, we can easily obtain

$$
\begin{aligned}
\left|e_{s k}(0)\right| & =\left|\lambda_{1} e_{1, k}(0)+\lambda_{2} e_{2, k}(0)+\cdots+e_{n, k}(0)\right| \\
& \leq \lambda_{1}\left|e_{1, k}(0)\right|+\lambda_{2}\left|e_{2, k}(0)\right|+\cdots+\left|e_{n, k}(0)\right| \\
& \leq \lambda_{1} \varepsilon_{1}+\lambda_{2} \varepsilon_{2}+\cdots+\varepsilon_{n}=\eta(0)
\end{aligned}
$$

which implies that $s_{k}(0)=e_{s k}(0)-\eta(0)\left(e_{s k}(0) / \eta(0)\right)=0$ is satisfied for any $k \in N$. For further use, we give the following equality:

$$
\begin{aligned}
s_{k} \operatorname{sat}\left(\frac{e_{s k}}{\eta(t)}\right) & = \begin{cases}0, & \text { if }\left|\frac{e_{s k}}{\eta(t)}\right| \leq 1 \\
s_{k} \operatorname{sgn}\left(e_{s k}\right), & \text { if }\left|\frac{e_{s k}}{\eta(t)}\right|>1\end{cases} \\
& =s_{k} \operatorname{sgn}\left(s_{k}\right)=\left|s_{k}\right|,
\end{aligned}
$$


where the sign function is defined as

$$
\operatorname{sgn}(\cdot)= \begin{cases}1, & \text { if } \cdot>0 \\ 0, & \text { if } \cdot=0 \\ -1, & \text { if } \cdot<0\end{cases}
$$

To continue the design procedure, we give the dynamic of $e_{k}$ as follows:

$$
\begin{aligned}
& \dot{e}_{i, k}=e_{i+1, k}+k_{i} z_{1, k}, \quad i=1, \ldots, n-1, \\
& \dot{e}_{n, k}=k_{n} z_{1, k}+v_{k}+\widehat{W}_{k}^{T} \phi\left(\widehat{x}_{k}\right)-y_{d}^{(n)} .
\end{aligned}
$$

Define a Lyapunov function as

$$
V_{s_{k}}=\frac{1}{2} s_{k}^{2}
$$

Taking the derivative of $V_{s_{k}}$ with respect to time yields

$$
\begin{aligned}
\dot{V}_{s_{k}}=s_{k} \dot{s}_{k}= \begin{cases}s_{k}\left(\dot{e}_{s k}-\dot{\eta}(t)\right), & \text { if } e_{s k}>\eta(t) \\
0, & \text { if }\left|e_{s k}\right| \leq \eta(t) \\
s_{k}\left(\dot{e}_{s k}+\dot{\eta}(t)\right), & \text { if } e_{s k}<-\eta(t)\end{cases} \\
=s_{k}\left(\dot{e}_{s k}-\dot{\eta}(t) \operatorname{sgn}\left(s_{k}\right)\right) \\
=s_{k}\left[\sum_{j=1}^{n-1}\left(\lambda_{j} e_{j+1, k}+\lambda_{j} k_{j} z_{1, k}\right)+K \eta(t) \operatorname{sgn}\left(s_{k}\right)\right. \\
\left.-y_{d}^{(n)}+k_{n} z_{1, k}+\widehat{W}_{k}^{T} \phi\left(\widehat{x}_{k}\right)+v_{k}\right] \\
=s_{k}\left[\sum_{j=1}^{n-1} \lambda_{j} e_{j+1, k}+\left[\Lambda^{T} 1\right] K_{0} z_{1, k}+K e_{s k}-y_{d}^{(n)}\right. \\
\left.+\widehat{W}_{k}^{T} \phi\left(\widehat{x}_{k}\right)+v_{k}\right]-K s_{k}^{2},
\end{aligned}
$$

where we utilize the following relation:

$$
\begin{aligned}
s_{k} & \left(-K e_{s k}+K \eta(t) \operatorname{sgn}\left(s_{k}\right)\right) \\
& =s_{k}\left(-K s_{k}-K \eta(t) \operatorname{sat}\left(\frac{e_{s k}}{\eta(t)}\right)+K \eta(t) \operatorname{sgn}\left(s_{k}\right)\right) \\
& =-K s_{k}^{2}-K \eta(t)\left|s_{k}\right|+K \eta(t)\left|s_{k}\right|=-K s_{k}^{2} .
\end{aligned}
$$

Choose the Lyapunov candidate for the whole closedloop system as $V_{k}=V_{z_{k}}+V_{U_{k}}+V_{s_{k}}$. Combining (27) and (35) we can obtain the derivative of $V_{k}$ as follows:

$$
\begin{aligned}
\dot{V}_{k} & \leq-\lambda_{\min }(Q)\left\|z_{k}\right\|^{2}-2 z_{k}^{T} P B \widetilde{W}_{k}^{T} \phi\left(\widehat{x}_{k}\right)+\frac{\lambda}{(1-\kappa)} \\
& \cdot \sum_{j=1}^{n} \rho_{j}^{2}\left(y_{k}\right)+\lambda D_{0}^{2}+4 \lambda l \varepsilon_{W}^{2}+\lambda \varepsilon_{0}^{2} \\
& +s_{k}\left[\sum_{j=1}^{n-1} \lambda_{j} e_{j+1, k}+\left[\begin{array}{ll}
\Lambda^{T} & 1
\end{array}\right] K_{0} z_{1, k}+K e_{s k}-y_{d}^{(n)}\right. \\
& \left.+\widehat{W}_{k}^{T} \phi\left(\widehat{x}_{k}\right)+v_{k}\right]-K s_{k}^{2} .
\end{aligned}
$$

For the convenience of expression, denote $\Xi\left(y_{k}\right) \triangleq(\lambda /(1-$ к)) $\sum_{j=1}^{n} \rho_{j}^{2}\left(y_{k}\right)+\lambda D_{0}^{2}+4 \lambda l \varepsilon_{W}^{2}+\lambda \varepsilon_{0}^{2}$; then (37) can be simplified as

$$
\begin{aligned}
\dot{V}_{k} & \leq-\lambda_{\min }(Q)\left\|z_{k}\right\|^{2}-2 z_{k}^{T} P B \widetilde{W}_{k}^{T} \phi\left(\widehat{x}_{k}\right) \\
& +s_{k}\left[\sum_{j=1}^{n-1} \lambda_{j} e_{j+1, k}+\left[\begin{array}{ll}
\Lambda^{T} & 1
\end{array}\right] K_{0} z_{1, k}+K e_{s k}-y_{d}^{(n)}\right. \\
& \left.+\widehat{W}_{k}^{T} \phi\left(\widehat{x}_{k}\right)+v_{k}+\frac{\Xi\left(y_{k}\right)}{s_{k}}\right]-K s_{k}^{2} .
\end{aligned}
$$

However, note that $\Xi\left(y_{k}\right) / s_{k}$ is not well defined at $s_{k}=$ 0 and may lead to controller singularity problem if we use $\Xi\left(y_{k}\right) / s_{k}$ in the controller design. In order to overcome this problem, we exploit the following property.

Lemma 14 (see [47]). For any constant $\eta>0$ and any variable $p \in R$,

$$
\lim _{p \rightarrow 0} \frac{\tanh ^{2}(p / \eta)}{p}=0 .
$$

Employing the hyperbolic tangent function, (38) becomes

$$
\begin{aligned}
\dot{V}_{k} & \leq-\lambda_{\min }(Q)\left\|z_{k}\right\|^{2}-2 z_{k}^{T} P B \widetilde{W}_{k}^{T} \phi\left(\widehat{x}_{k}\right) \\
& +s_{k}\left[\sum_{j=1}^{n-1} \lambda_{j} e_{j+1, k}+\left[\begin{array}{ll}
\Lambda^{T} & 1
\end{array}\right] K_{0} z_{1, k}+K e_{s k}-y_{d}^{(n)}\right. \\
& \left.+\widehat{W}_{k}^{T} \phi\left(\widehat{x}_{k}\right)+v_{k}+\frac{b \tanh ^{2}\left(s_{k} / \eta(t)\right) \Xi\left(y_{k}\right)}{s_{k}}\right] \\
& +\left[1-b \tanh ^{2}\left(\frac{s_{k}}{\eta(t)}\right)\right] \Xi\left(y_{k}\right)-K s_{k}^{2} .
\end{aligned}
$$

From Lemma 14, we know that $\lim _{s_{k} \rightarrow 0} b \tanh ^{2}\left(s_{k} /\right.$ $\eta(t)) \Xi\left(y_{k}\right) / s_{k}=0$. Hence, $b \tanh ^{2}\left(s_{k} / \eta(t)\right) \Xi\left(y_{k}\right) / s_{k}$ is defined at $s_{k}=0$ and the problem of possible singularity is avoided. 
Apparently, $(\lambda /(1-\kappa)) \sum_{j=1}^{n} \rho_{j}^{2}\left(y_{k}\right)$ is continuous and welldefined over compact set $\Omega_{y}=\left\{y_{k}\right\} \subset R$, so it can be approximated by a time-invariant neural network to arbitrary accuracy as $(\lambda /(1-\kappa)) \sum_{j=1}^{n} \rho_{j}^{2}\left(y_{k}\right)=W_{\Xi}^{* T} \phi_{\Xi}\left(y_{k}\right)+\varepsilon_{\Xi}\left(y_{k}\right)$, where $\left|\varepsilon_{\Xi}\left(y_{k}\right)\right| \leq \bar{\varepsilon}_{\Xi}$ is the approximation error, $W_{\Xi}^{*} \in R^{l_{\Xi}}$ are unknown ideal time-invariant weight vector, and $\phi_{\Xi}\left(y_{k}\right) \epsilon$ $R^{l_{\Xi}}$ is the basis functions, where $l_{\Xi}$ denotes the NN node number. Also, we make the following finiteness assumption for $W_{\Xi}^{*}$.

Assumption 15. The ideal weight vector $W_{\Xi}^{*}$ is bounded; that is,

$$
\left\|W_{\Xi}^{*}\right\| \leq \varepsilon_{W_{\Xi}}
$$

where $\varepsilon_{W_{\Xi}}$ is unknown positive constant.

Then $\Xi\left(y_{k}\right)$ can be expressed as

$$
\begin{aligned}
\Xi\left(y_{k}\right)= & W_{\Xi}^{* T} \phi_{\Xi}\left(y_{k}\right)+\varepsilon_{\Xi}\left(y_{k}\right)+\lambda D_{0}^{2}+4 \lambda l \varepsilon_{W}^{2} \\
& +\lambda \varepsilon_{0}^{2} .
\end{aligned}
$$

For convenience of presentation, denote $\mu \triangleq \varepsilon_{\Xi}\left(y_{k}\right)+$ $\lambda D_{0}^{2}+4 \lambda l \varepsilon_{W}^{2}+\lambda \varepsilon_{0}^{2}$. Furthermore, we rewrite $\Xi\left(y_{k}\right)$ as $\Xi\left(y_{k}\right)=$ $W_{2}^{* T} \phi_{2}\left(y_{k}\right)$, with $W_{2}^{*}=\left[W_{\Xi}^{* T}, \mu\right]^{T}$ and $\phi_{2}\left(y_{k}\right)=\left[\phi_{\Xi}^{T}\left(y_{k}\right), 1\right]^{T}$.

Until now, we can design the output feedback controller as follows:

$$
\begin{aligned}
v_{k}= & -\sum_{j=1}^{n-1} \lambda_{j} e_{j+1, k}-\left[\begin{array}{ll}
\Lambda^{T} & 1
\end{array}\right] K_{0} z_{1, k}-K e_{s k}+y_{d}^{(n)} \\
& -\widehat{W}_{k}^{T}(t) \phi\left(\widehat{x}_{k}\right) \\
& -\frac{b \widehat{W}_{2, k}^{T} \phi_{2}\left(y_{k}\right) \tanh ^{2}\left(s_{k} / \eta(t)\right)}{s_{k}}
\end{aligned}
$$

where $\widehat{W}_{k}(t)$ and $\widehat{W}_{2, k}$ are the estimates of $W^{*}(t)$ and $W_{2}^{*}$, respectively.

The difference type and differential-difference type update algorithms are designed, respectively, for $\widehat{W}_{k}(t)$ and $\widehat{W}_{2, k}$ as follows:

$$
\begin{aligned}
& \widehat{W}_{k}(t)=\widehat{W}_{k-1}(t)+2 q_{1} z_{k}^{T} P B \phi\left(\widehat{x}_{k}\right), \\
& \widehat{W}_{0}(t)=0,
\end{aligned}
$$

$$
t \in[0, T]
$$

$$
\begin{aligned}
(1-\gamma) \dot{\widehat{W}}_{2, k}= & -\gamma \widehat{W}_{2, k}+\gamma \widehat{W}_{2, k-1} \\
& +q_{2} b \tanh ^{2}\left(\frac{s_{k}}{\eta(t)}\right) \phi_{2}\left(y_{k}\right), \\
\widehat{W}_{2, k}(0)= & \widehat{W}_{2, k-1}(T), \\
\widehat{W}_{2,0}(t)= & 0,
\end{aligned}
$$

$$
t \in[0, T]
$$

where $q_{1}, q_{2}>0$ and $0<\gamma<1$ are design parameters.
Define the estimation errors as $\widetilde{W}_{k}(t)=\widehat{W}_{k}(t)-W^{*}(t)$ and $\widetilde{W}_{2, k}=\widehat{W}_{2, k}-W_{2, k}^{*}$. Then, substituting the controller (43) back into (40) yields

$$
\begin{aligned}
\dot{V}_{k} \leq & -\lambda_{\min }(Q)\left\|z_{k}\right\|^{2}-2 z_{k}^{T} P B \widetilde{W}_{k}^{T} \phi\left(\widehat{x}_{k}\right) \\
& -b \widetilde{W}_{2, k}^{T} \phi_{2}\left(y_{k}\right) \tanh ^{2}\left(\frac{s_{k}}{\eta(t)}\right) \\
& +\left[1-b \tanh ^{2}\left(\frac{s_{k}}{\eta(t)}\right)\right] \Xi\left(y_{k}\right)-K s_{k}^{2} .
\end{aligned}
$$

For subsequent discussion, we rewrite (46) as

$$
\begin{aligned}
2 z_{k}^{T} P B \widetilde{W}_{k}^{T} \phi\left(\hat{x}_{k}\right)+b \widetilde{W}_{2, k}^{T} \phi_{2}\left(y_{k}\right) \tanh ^{2}\left(\frac{s_{k}}{\eta(t)}\right) \\
\leq-\dot{V}_{k}-\lambda_{\min }(Q)\left\|z_{k}\right\|^{2} \\
+\left[1-b \tanh ^{2}\left(\frac{s_{k}}{\eta(t)}\right)\right] \Xi\left(y_{k}\right)-K s_{k}^{2} .
\end{aligned}
$$

For further analysis, we exploit the following property of the tangent hyperbolic function.

Lemma 16. Consider the set $\Omega_{s_{k}}$ defined by $\Omega_{s_{k}}:=\left\{s_{k}|| s_{k} \mid \leq\right.$ $m \eta(t)\}$. Then, for any $s_{k} \notin \Omega_{s_{k}}$, the following inequality is satisfied:

$$
1-b \tanh ^{2}\left(\frac{s_{k}}{\eta(t)}\right)<0,
$$

where $b>1, m=\ln (\sqrt{b /(b-1)}+\sqrt{1 /(b-1)})$.

Proof. See Appendix A.

The convergence and boundedness property of the proposed observer-based AILC scheme are summarized in the following theorem.

Theorem 17. Considering the closed-loop system consisting of plant (1) and input saturation model (3) under Assumptions 15, 11, 12 and 15, design state observer (15) and adaptive iterative learning controller (43) with parameter update algorithms (44) and (45); the following properties can be guaranteed: (i) all the signals of the closed-loop system are bounded in $L_{T}^{\infty}$-norm; (ii) the error $e_{s k}(t)$ converges to a small region around zero as $k \rightarrow$ $\infty$; that is, $\lim _{k \rightarrow \infty} \int_{0}^{T}\left(e_{s k}(\sigma)\right)^{2} \mathrm{~d} \sigma \leq \varepsilon_{\text {esk }}, \varepsilon_{\text {esk }}=(1 / 2 K)(1+$ $m)^{2} \varepsilon^{2}$; and (iii) transient performance $\lim _{k \rightarrow \infty}\left|y_{k}(t)-y_{d}(t)\right| \leq$ $\min \left\{k_{0} \sum_{i=1}^{n-1} \sqrt{\varepsilon_{i}^{2}} e^{-\lambda_{0} t}+(1+m) \varepsilon k_{0}\left(1 /\left(\lambda_{0}-K\right)\right)\left(e^{-K t}-e^{-\lambda_{0} t}\right)\right.$, $\left.\left[p_{1}+\left(1 / k_{1}\right)\left(K p_{1}+p_{2}\right)\right] \varepsilon e^{-K t}\right\}$, where $\lambda_{0}, k_{0}, p_{1}$, and $p_{2}$ are positive constants and will be given later.

Proof. See Appendix B.

\section{Simulation Study}

In this section, we present a simulation example to verify the effectiveness of proposed control scheme. Consider the 
following second-order nonlinear time delay system with input saturation:

$$
\begin{aligned}
\dot{x}_{1, k}(t) & =x_{2, k}(t), \\
\dot{x}_{2, k}(t) & =f\left(x_{k}(t), t\right)+h\left(x_{\tau, k}(t), t\right)+u_{k}(t)+d(t), \\
y_{k}(t) & =x_{1, k}(t),
\end{aligned}
$$

where $f\left(X_{k}(t), t\right)=-x_{1, k}(t) x_{2, k}(t) \sin \left(x_{1, k}(t) x_{2, k}(t)\right)$ and $h\left(y_{\tau, k}, t\right)=0.5 \sin (t) e^{-|\cos (0.5 t)|}\left(y_{\tau_{1}} \sin \left(y_{\tau_{1}}\right)+y_{\tau_{2}} \sin \left(y_{\tau_{2}}\right)\right)$, with $\tau_{1}=0.5(1+\sin (0.3 t))$ and $\tau_{2}=0.8(1-\sin (0.5 t))$. The external disturbance is given by $d=0.1 * \operatorname{rand} * \sin t$, where rand presents Gaussian noise which takes a random value on $[0,1]$. We choose $Q=\left[\begin{array}{cc}0.001 & 0 \\ 0 & 0.002\end{array}\right]$. By using LMI toolbox, we can obtain $K_{0}=[3.2894,2.9764]^{T}$ and $P=\left[\begin{array}{ccc}0.4741 & -0.2848 \\ -0.2848 & 0.4741\end{array}\right]$. Next, we give the simulation study in three cases.

Case 1. The desired trajectory is given by $x_{d}(t)=$ $[\sin t, \cos t]^{T}$. The systems run on $[0,4 \pi]$ repetitively. The design parameters are chosen as $\varepsilon_{1}=\varepsilon_{2}=1, \lambda=2$, $K=2, \gamma=0.5, q_{1}=0.5, q_{2}=1, \varepsilon=\lambda \varepsilon_{1}+\varepsilon_{2}=3$, and $u_{M}=1.3$. The parameters for two NNs are chosen as $l=30$, $\mu_{j}=(1 / l)(2 j-l)[2,3,2,3,3], \sigma_{j}=1.5, j=1,2, \ldots, l$, and $l_{\Xi}=15, \mu_{\Xi j}=\left(2 / l_{\Xi}\right)\left(2 j-l_{\Xi}\right), \sigma_{\Xi j}=1, j=1,2, \ldots, l_{\Xi}$. The upper bound of control input is $u_{M}=1$.3. Parts of the simulation results are shown in Figures 2-9. Figures 2 and 6 express the trajectories of the observer output and the desired reference signal of the first iteration and the tenth iteration, respectively; Figures 3 and 7 show the trajectories of $x_{1, k}$ and $\hat{x}_{1, k}$ of the first iteration $(k=1)$ and the tenth iteration $(k=10)$, respectively; Figures 4 and 7 show the effects of input saturation. Obviously, these signals are bounded. Figures 8 and 9 show the convergence of $\int_{0}^{T} z_{1, k}^{2}(t) \mathrm{d} t$ and $\int_{0}^{T} s_{k}^{2}(t) \mathrm{d} t$ along the iteration axis, which indicates that the proposed approach achieves perfect tracking by iterative learning.

Case 2. To show the control performance for more complicated desired trajectory, we choose the desired trajectory as $x_{d}(t)=[\sin t+\sin (0.5 t), \cos t+0.5 \cos (0.5 t)]^{T}$. The system runs on $[0,8 \pi]$ repetitively. The design parameters are chosen as the same as Case 1 . The control input is bounded by $u_{M}=$ 4. The systems run for ten times repeatedly. Parts of the simulation results are shown in Figures 10-17. Figures 1012 show the results of the first iteration $(k=1)$. It can be seen that the tracking performance of the first iteration is not so good. Figures $13-15$ present the simulation results of the tenth iteration $(k=10)$. Obviously, the tracking performance has been improved greatly compared with that of the first iteration, which can be also demonstrated by convergence of $\int_{0}^{T} z_{1, k}^{2}(t) \mathrm{d} t$ and $\int_{0}^{T} s_{k}^{2}(t) \mathrm{d} t$ along the iteration domain in Figures 16 and 17. The results indicate the validity of the proposed method for more complicated design trajectory.

Case 3. Finally, the contribution of the proposed observerbased AILC scheme is shown by comparing the proposed controller with traditional adaptive neural network controller

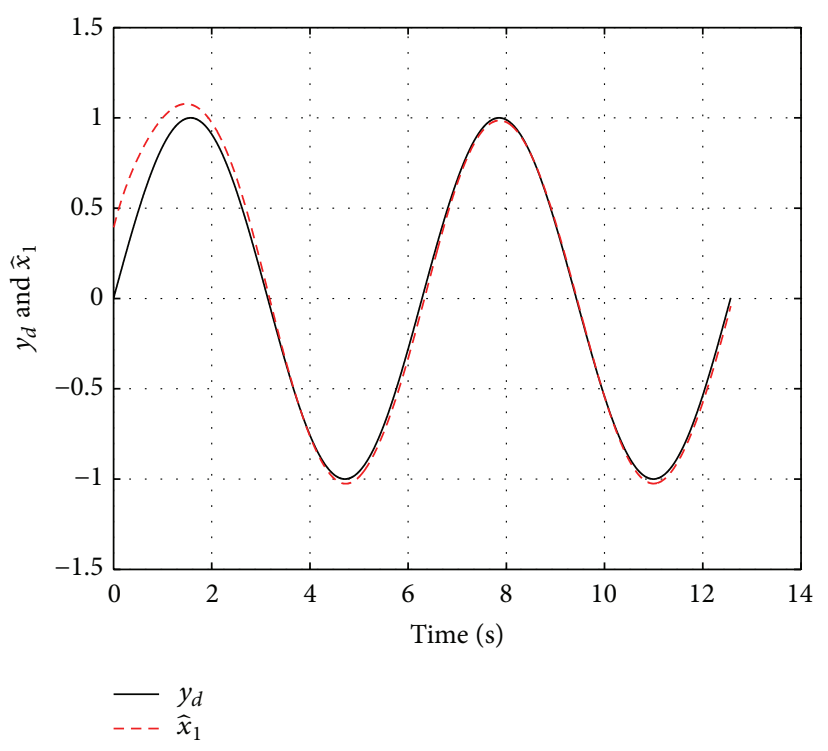

FIGURE 2: Reference signal $y_{d}$ on observer state $\widehat{x}_{1, k}(k=1)$ in Case 1.

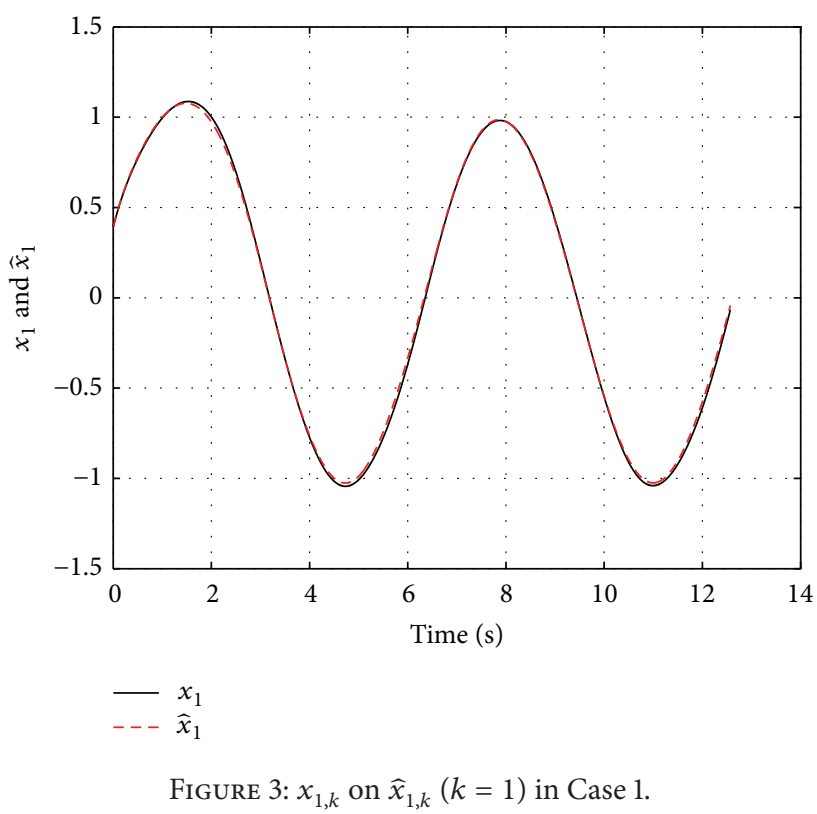

[48]. The controller is the same, but the adaptive laws using $\sigma$-modification for adaptive laws of parameters are given by

$$
\begin{aligned}
\dot{\widehat{W}} & =\Gamma_{W}\left(z_{k}^{T} P B \phi\left(\widehat{x}_{k}\right)-\delta_{W} \widehat{W}\right), \\
\dot{W}_{2} & =q_{2} s_{k} \phi_{2}\left(y_{k}\right)-\gamma \widehat{W}_{2} .
\end{aligned}
$$

The design parameters are chosen as $\Gamma_{W}=\operatorname{diag}\{0.2, \ldots$, $0.2\}, \delta_{W}=0.5, q_{2}=1, \gamma=0.5$, and $u_{M}=3.5$. Since traditional adaptive NN controller runs in time domain, the notation $k$ in this case does not have any practical meaning. Figures 18-20 provide simulation results. From the simulation results shown in Figures 18-20, it is obvious that the adaptive NN 


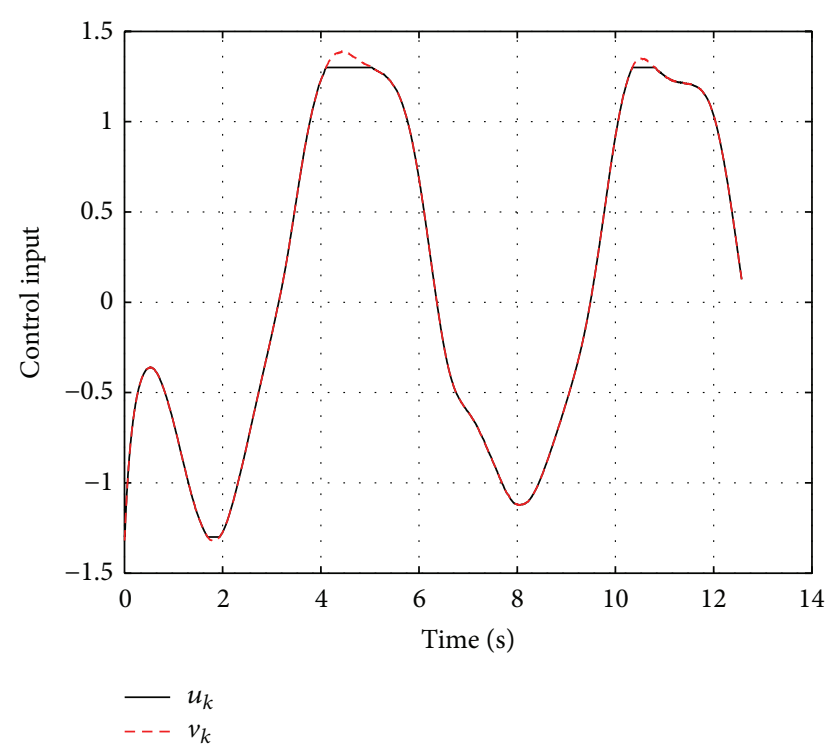

FIgURE 4: Control output $u_{k}$ and input $v_{k}(k=1)$ in Case 1.

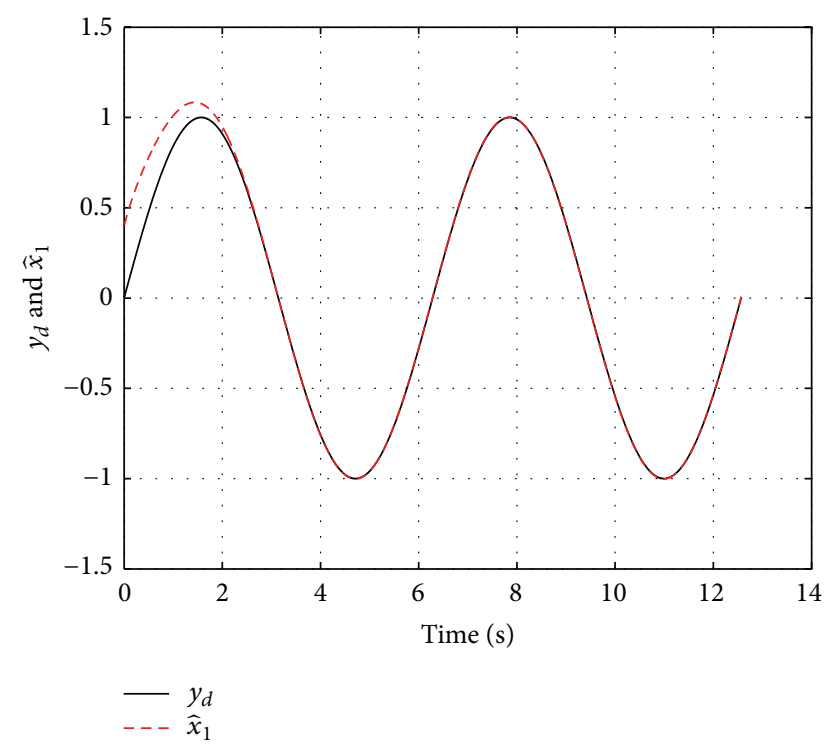

FIgURE 5: Reference signal $y_{d}$ on observer state $\widehat{x}_{1, k}(k=10)$ in Case 1.

controller is unable to achieve perfect tracking performance and performs much worse than the proposed approach.

According to the simulation results, it is confirmed that the proposed AILC can guarantee fairly good control performance for uncertain nonlinear systems with unknown timevarying delays and control input saturation in the presence of external disturbance. Moreover, it is verified that our control scheme is more suitable than robust adaptive neural network control methods for finite time repeated problem.

\section{Conclusions}

An adaptive iterative learning control scheme has been proposed for a class of nonlinear time-varying systems
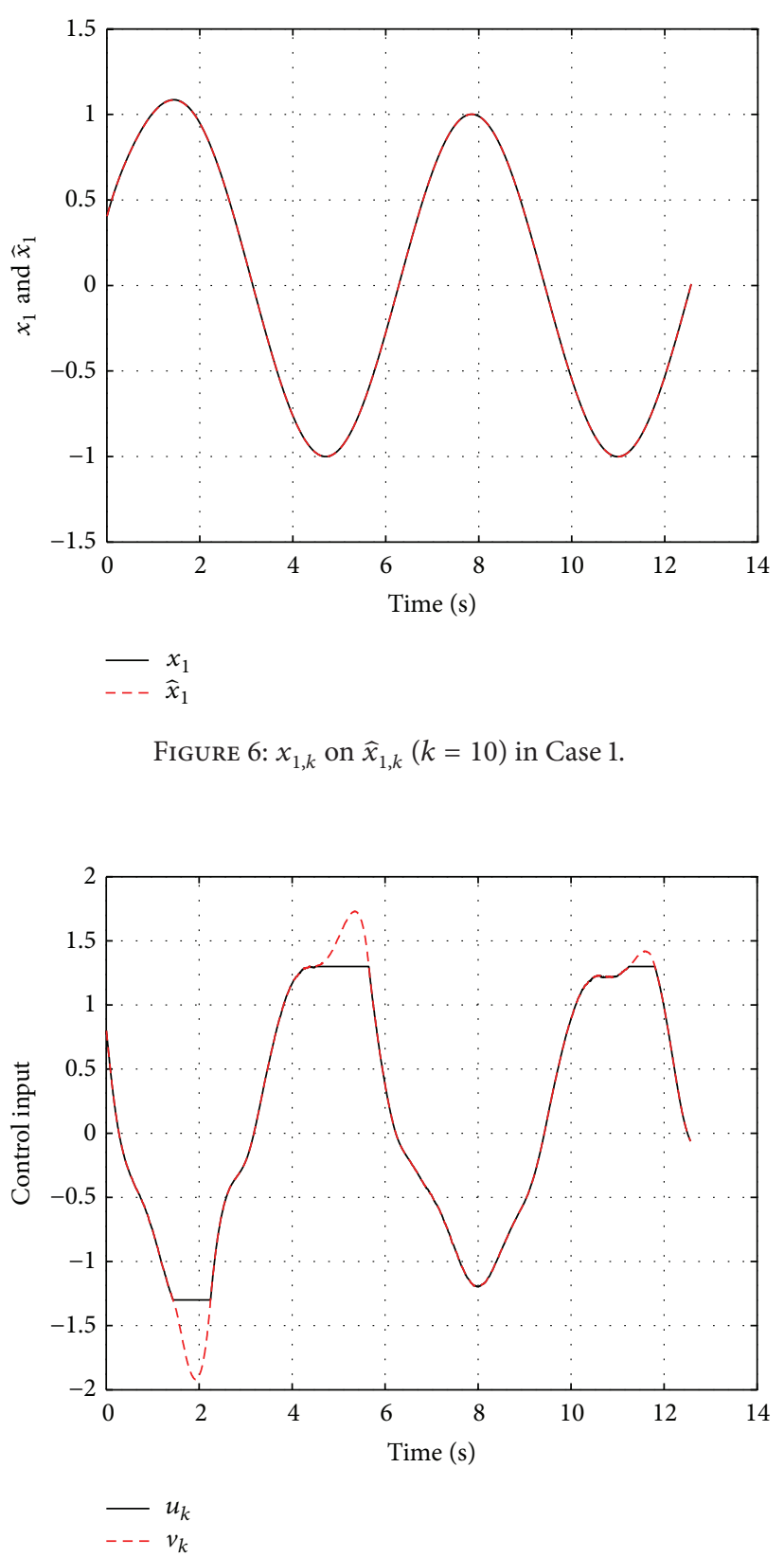

Figure 7: Control output $u_{k}$ and input $v_{k}(k=10)$ in Case 1.

with unknown time-varying delays and input saturation running on a finite time interval repeatedly. An observer has been designed to estimate the states. The uncertainties from unknown time-varying delays have been compensated for by using appropriate Lyapunov-Krasovskii functional in the Lyapunov function candidate, such that control law is delay-independent. Both time-varying and time-invariant RBF neural networks are employed to deal with the systems uncertainties. Theoretical analysis by constructing Lyapunovlike CEF has shown that the proposed systematic design is able to guarantee the convergence of tracking errors to a small residual domain around the origin and the boundedness of all the closed-loop signals. Simulation results have been 


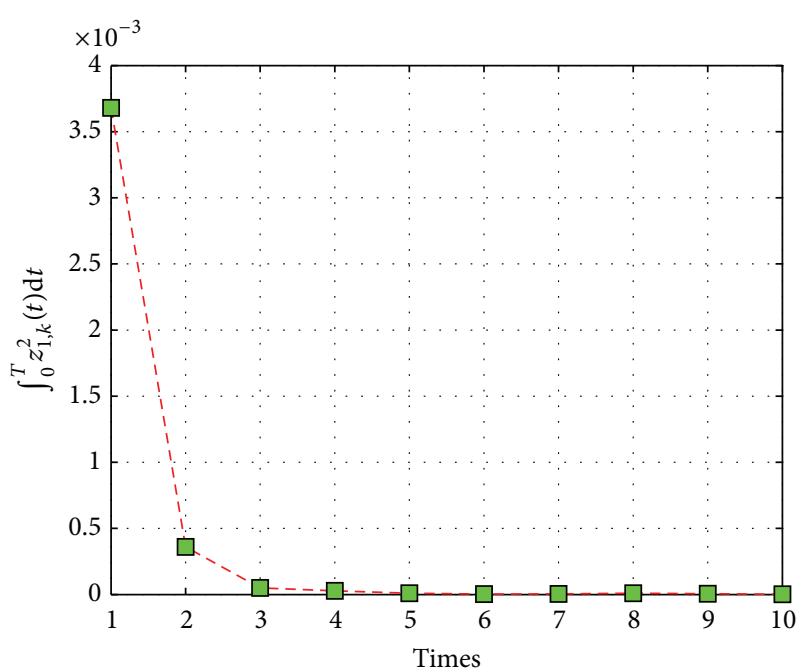

FIgURE 8: $\int_{0}^{T} z_{1, k}^{2}(t) \mathrm{d} t$ versus the number of iterations in Case 1.

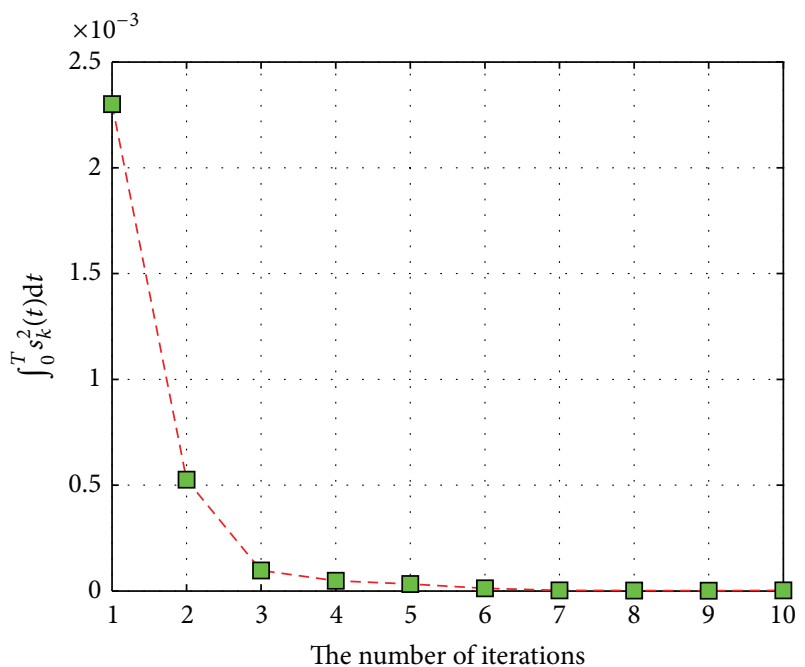

Figure 9: $\int_{0}^{T} s_{k}^{2}(t) \mathrm{d} t$ versus the number of iterations in Case 1.

provided to show the effectiveness the proposed control scheme.

\section{Appendices}

\section{A. Proof of Lemma 16}

Denote $x=s_{k}(t) / \eta(t)$. The inequality (48) in Lemma 16 can be expressed as

$$
\frac{1}{b}<\tanh ^{2}(x)=\left(\frac{e^{x}-e^{-x}}{e^{x}+e^{-x}}\right)^{2}=1-\left(\frac{2}{e^{x}+e^{-x}}\right)^{2} .
$$

Since $e^{x}$ and $e^{-x}$ are positive, we can know

$$
e^{x}+e^{-x}>2 \sqrt{\frac{b}{b-1}} .
$$

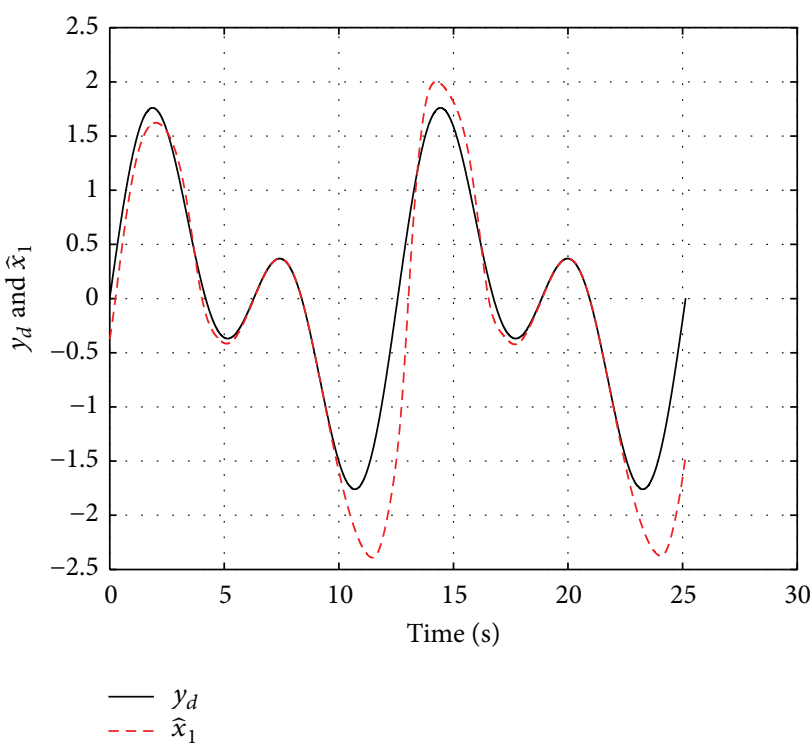

FIGURE 10: Reference signal $y_{d}$ on observer state $\widehat{x}_{1, k}(k=1)$ in Case 2.

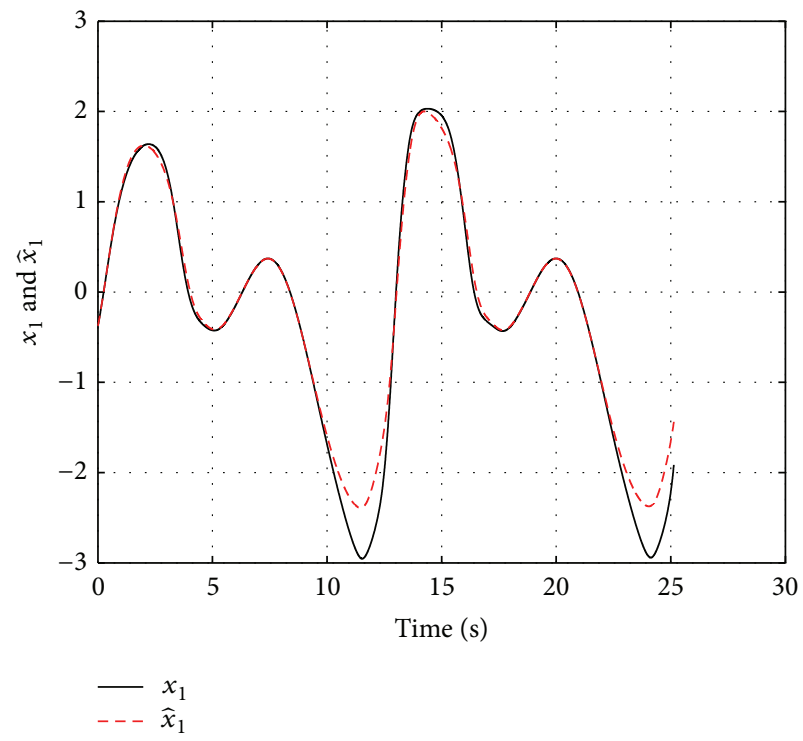

FIGURE 11: $x_{1, k}$ on $\widehat{x}_{1, k}(k=1)$ in Case 2 .

Further, we can obtain

$$
e^{2 x}-2 \sqrt{\frac{b}{b-1}} e^{x}+1>0
$$

Solving quadratic inequality (A.3), we get

$$
0<e^{x}<\sqrt{\frac{b}{(b-1)}}-\sqrt{\frac{1}{(b-1)}}
$$

$$
\text { or } e^{x}>\sqrt{\frac{b}{(b-1)}}+\sqrt{\frac{1}{(b-1)}} \text {. }
$$




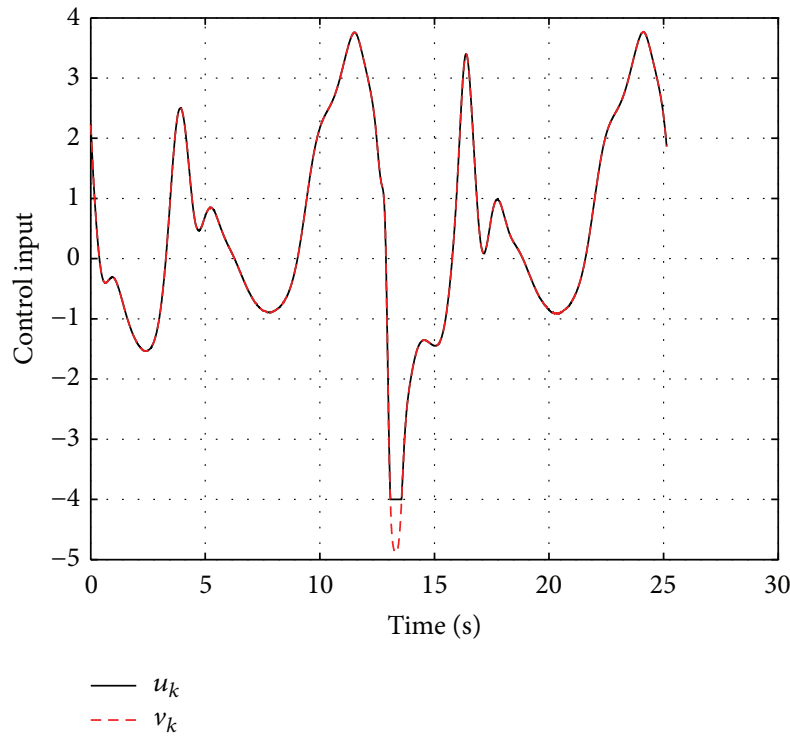

FIGURE 12: Control output $u_{k}$ and input $v_{k}(k=1)$ in Case 2.

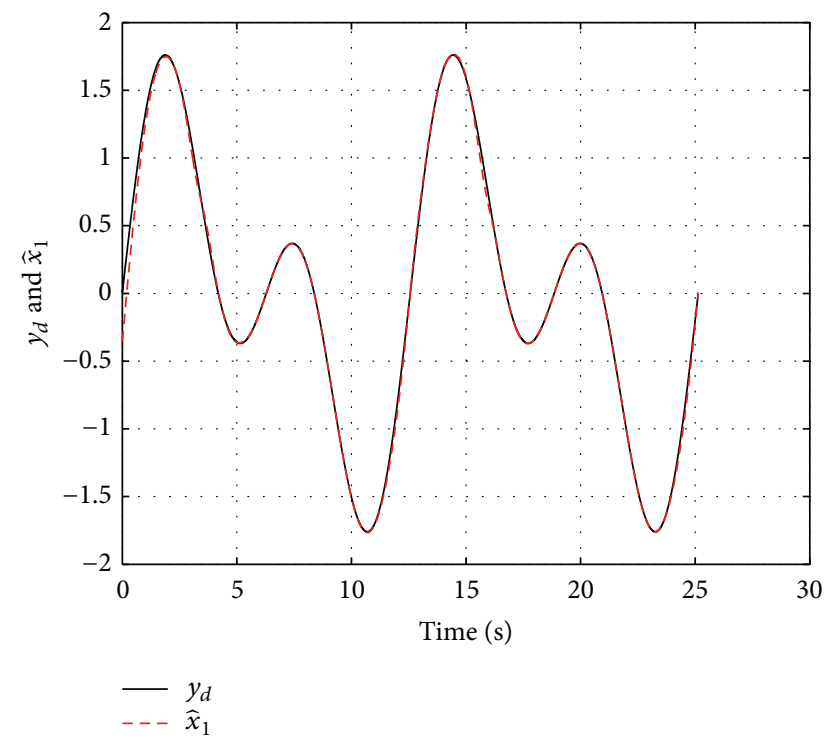

FIGURE 13: Reference signal $y_{d}$ on observer state $\widehat{x}_{1, k}(k=10)$ in Case 2.

On the other hand, from $\left|s_{k}(t)\right|>m \eta(t)$, we know

$$
x<-m
$$

or $x>m$

which implies

$$
\begin{aligned}
0 & <e^{x}<\frac{1}{\sqrt{b /(b-1)}+\sqrt{1 /(b-1)}} \\
& =\sqrt{\frac{b}{(b-1)}}-\sqrt{\frac{1}{(b-1)}}
\end{aligned}
$$

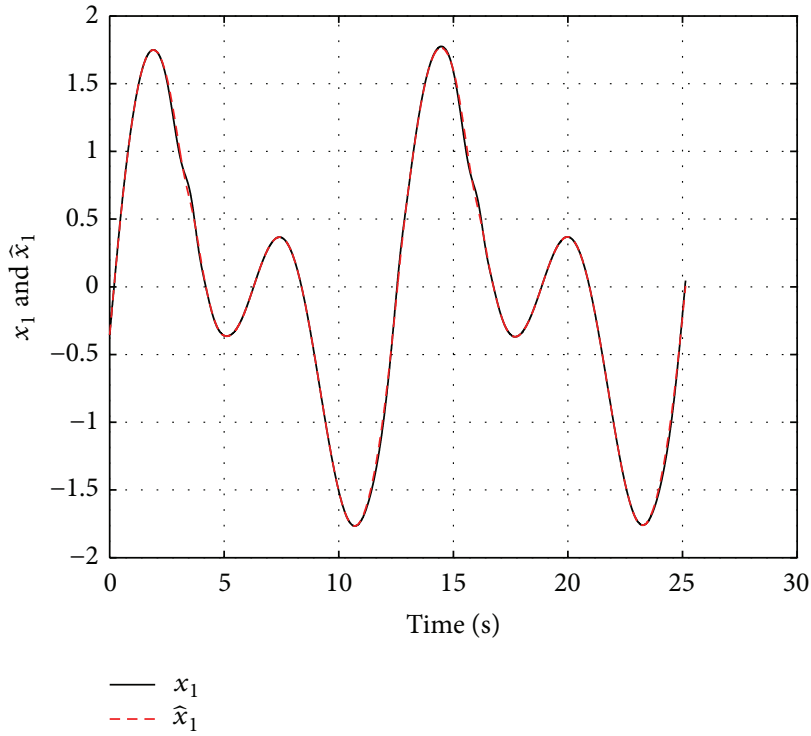

Figure 14: $x_{1, k}$ on $\widehat{x}_{1, k}(k=10)$ in Case 2.

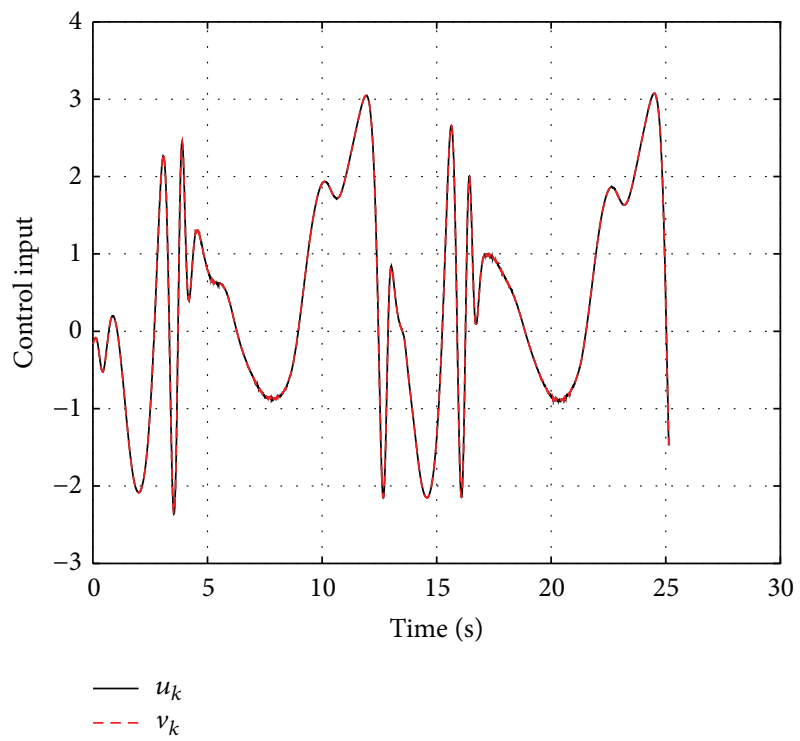

FIGURE 15: Control output $u_{k}$ and input $v_{k}(k=10)$ in Case 2.

$$
\text { or } e^{x}>\sqrt{\frac{b}{(b-1)}}+\sqrt{\frac{1}{(b-1)}} \text {. }
$$

Obviously, Lemma 16 holds.

\section{B. Proof of Theorem 17}

The term $\left[1-b \tanh ^{2}\left(s_{k} / \eta(t)\right)\right] \Xi\left(y_{k}\right)$ in (47) may be positive or negative, which depends on the size of $s_{k}$. Thus, two cases must be considered for subsequent analysis of stability.

Case $1\left(s_{k} \in \Omega_{s_{k}}\right)$. If $s_{k} \in \Omega_{s_{k}}$, then $\left|s_{k}\right| \leq m \eta(t)$ is satisfied. We divide it into three cases. (1) If $s_{k}=0$, we know that $e_{s k}$ is 


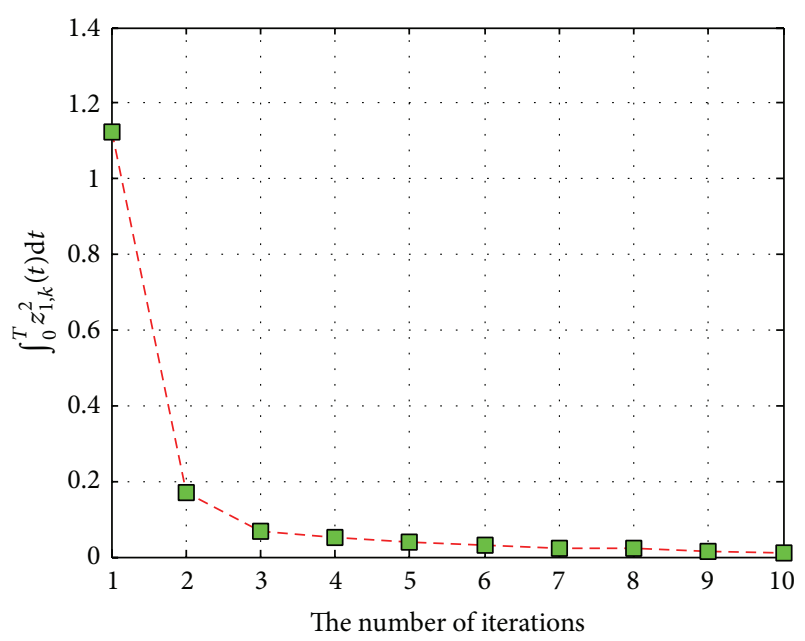

FIGURE 16: $\int_{0}^{T} z_{1, k}^{2}(t) \mathrm{d} t$ versus the number of iterations in Case 2.

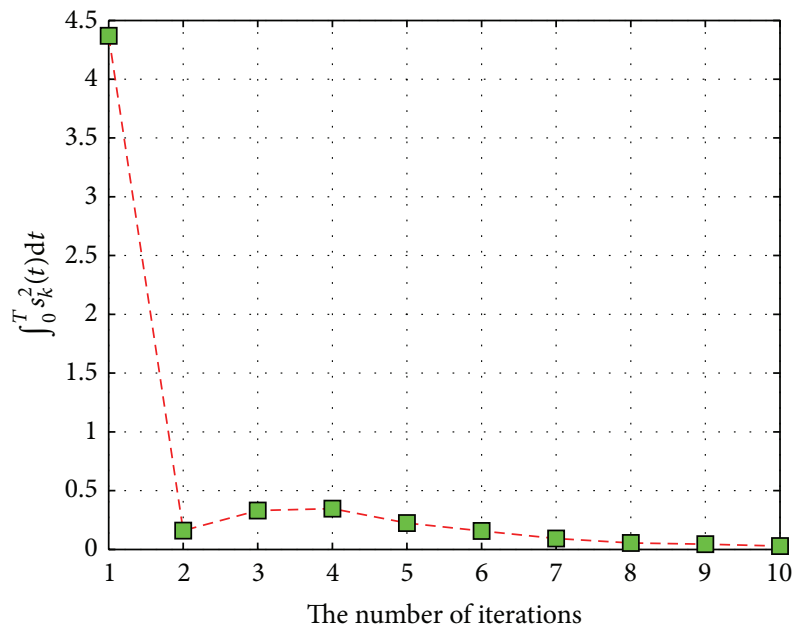

FIGURE 17: $\int_{0}^{T} s_{k}^{2}(t) \mathrm{d} t$ versus the number of iterations in Case 2.

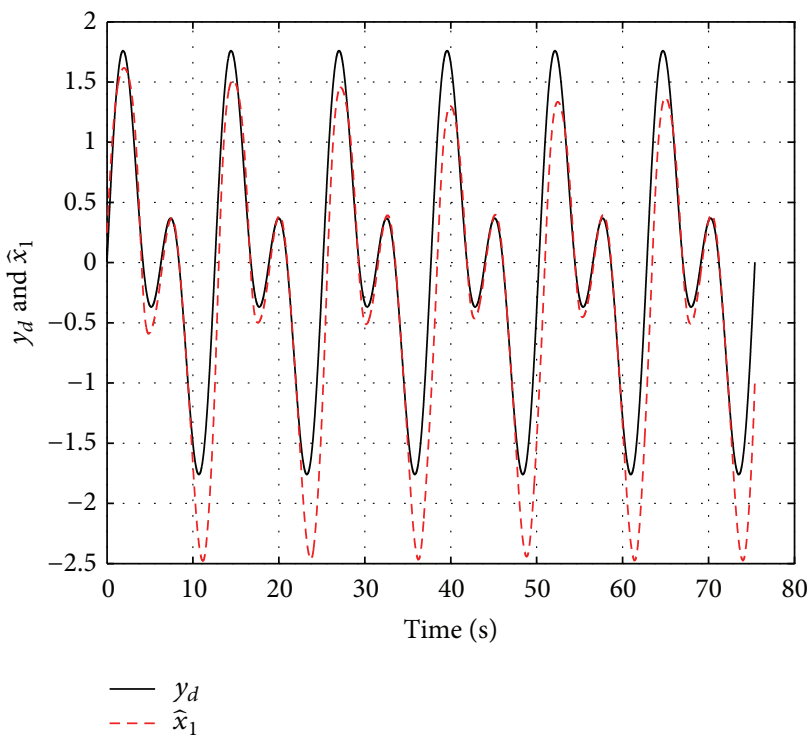

FIGURE 18: Reference signal $y_{d}$ on observer state $\widehat{x}_{1}$ in Case 3.

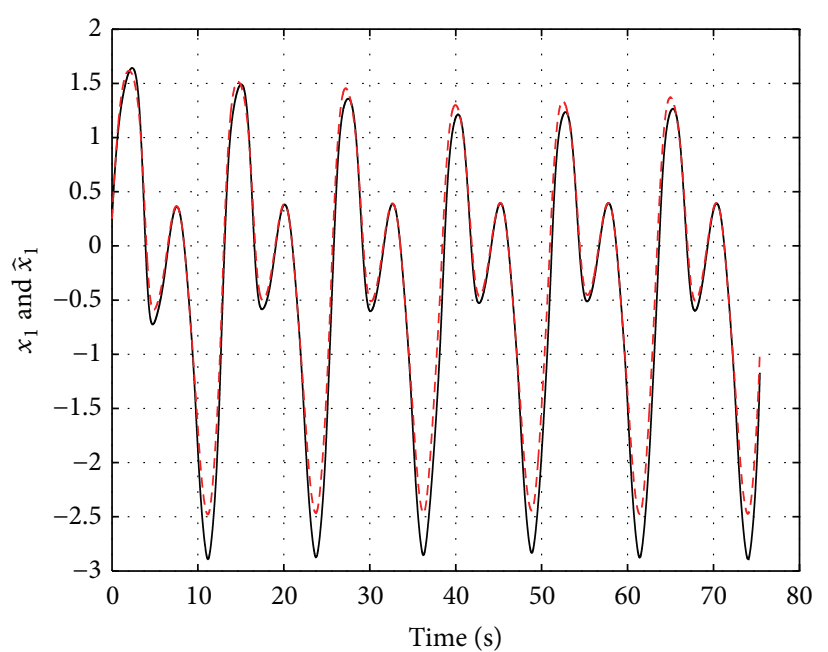

$\begin{array}{ll}- & x_{1} \\ --- & \widehat{x}_{1}\end{array}$

Figure 19: $x_{1}$ on $\widehat{x}_{1}$ in Case 3 .

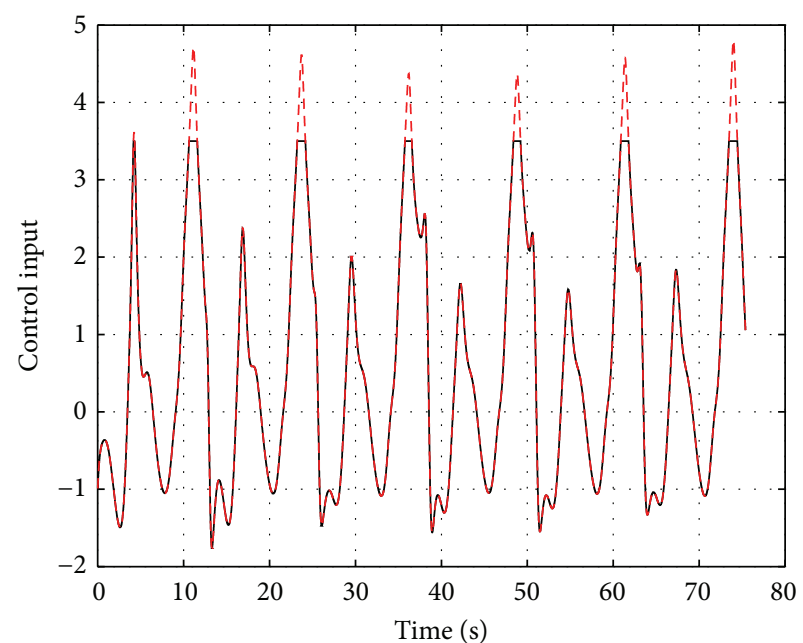

$-u_{k}$

Figure 20: Control output $u_{k}$ and input $v_{k}$ in Case 3.

bounded by $\eta(t)$; that is, $\left|e_{s k}\right| \leq \eta(t)$; (2) if $s_{k}>0$, we know $s_{k}=e_{s k}-\eta(t)$; then from $\left|s_{k}\right| \leq m \eta(t)$ we can obtain $s_{k}=$ $e_{s k}-\eta(t) \leq m \eta(t)$ which further implies $0<e_{s k} \leq(1+m) \eta(t)$; (3) similarly, if $s_{k}<0$, we have $s_{k}=e_{s k}+\eta(t) \geq-m \eta(t)$ which implies $0>e_{s k} \geq-(1+m) \eta(t)$. Synthesizing the foregoing discussion we know that $\left|e_{s k}\right| \leq(1+m) \eta(t)$ holds. Thus it implies the boundedness of $\widehat{x}_{i, k}$ since $x_{d}(t)$ is bounded in $L_{T}^{\infty}$-norm. From updating laws (44) and (45), we know that $\widehat{W}_{k}(t) \in L_{T}^{\infty}$ and $\widehat{W}_{2, k}(t) \in L_{T}^{\infty}$. Following this chain of reasoning, the boundedness of $z_{k}$ and $x_{k}$ in $L_{T}^{\infty}$-norm can be deduced. Then, we can achieve the boundedness of $v_{k}$. As such, all closed-loop signals are bounded $L_{T}^{\infty}$-norm. Furthermore, from $\left|e_{s k}\right| \leq(1+m) \eta(t)$ and the definition of $e_{k}$, it can be easily derived that $e_{1, k}$ and $e_{2, k}$ are in a small 
ball of $p_{1} \eta(t)$ and $p_{2} \eta(t)$, respectively, with $p_{1}$ and $p_{2}$ as small positive constants, which, in other words, are $\left|e_{1, k}\right| \leq p_{1} \eta(t)$ and $\left|e_{2, k}\right| \leq p_{2} \eta(t)$. Then it can be derived that $\left|\dot{e}_{1, k}\right| \leq$ $p_{1} K \eta(t)$. From (33), it follows that

$$
k_{1}\left|z_{1, k}\right| \leq\left|e_{2, k}\right|+\left|\dot{e}_{1, k}\right|=\left(K p_{1}+p_{2}\right) \varepsilon e^{-K t} .
$$

Then it implies that

$$
\begin{aligned}
\left|y_{k}-y_{d}\right| & =\left|z_{1, k}+e_{1, k}\right| \leq\left|z_{1, k}\right|+\left|e_{1, k}\right| \\
& \leq\left[p_{1}+\frac{1}{k_{1}}\left(K p_{1}+p_{2}\right)\right] \varepsilon e^{-K t} .
\end{aligned}
$$

In this case, it is obvious that all the signals of the closed system are bounded.

Case $2\left(s_{k}(t) \notin \Omega_{s_{k}}\right)$. According to Lemma 16, it is clear that $\left[1-b \tanh ^{2}\left(s_{k} / \eta(t)\right)\right] \Xi\left(y_{k}\right)$ can be removed from the righthand side of (47). Therefore, it follows from (47) that

$$
\begin{aligned}
& 2 z_{k}^{T} P B \widetilde{W}_{k}^{T} \phi\left(\widehat{x}_{k}\right)+b \widetilde{W}_{2, k}^{T} \phi_{2}\left(y_{k}\right) \tanh ^{2}\left(\frac{s_{k}}{\eta(t)}\right) \\
& \leq-\dot{V}_{k}-\lambda_{\min }(Q)\left\|z_{k}\right\|^{2}-K s_{k}^{2} .
\end{aligned}
$$

Next, we will check the stability of the system by using CEF-based analysis. Define the Lyapunov-like CEF as follows:

$$
\begin{aligned}
E_{k}(t)= & \frac{1}{2 q_{1}} \int_{0}^{t} \widetilde{W}_{k}^{T} \widetilde{W}_{k} \mathrm{~d} \sigma+\frac{\gamma}{2 q_{2}} \int_{0}^{t} \widetilde{W}_{2, k}^{T} \widetilde{W}_{2, k} \mathrm{~d} \sigma \\
& +\frac{(1-\gamma)}{2 q_{2}} \widetilde{W}_{2, k}^{T} \widetilde{W}_{2, k} .
\end{aligned}
$$

The subsequent derivations consist of five parts.

(1) Difference of $E_{k}(t)$. Computing the difference of $E_{k}(t)$, we obtain

$$
\begin{aligned}
\Delta E_{k}(t)= & E_{k}(t)-E_{k-1}(t) \\
= & \frac{1}{2 q_{1}} \int_{0}^{t}\left[\widetilde{W}_{k}^{T} \widetilde{W}_{k}-\widetilde{W}_{k-1}^{T} \widetilde{W}_{k-1}\right] \mathrm{d} \sigma \\
& +\frac{\gamma}{2 q_{2}} \int_{0}^{t}\left[\widetilde{W}_{2, k}^{T} \widetilde{W}_{2, k}-\widetilde{W}_{2, k-1}^{T} \widetilde{W}_{2, k-1}\right] \mathrm{d} \sigma \\
& +\frac{(1-\gamma)}{2 q_{2}}\left[\widetilde{W}_{2, k}^{T} \widetilde{W}_{2, k}-\widetilde{W}_{2, k-1}^{T} \widetilde{W}_{2, k-1}\right] .
\end{aligned}
$$

Recalling difference type adaptive learning law (44) and utilizing the algebraic relation $(a-b)^{T}(a-b)-(a-c)^{T}(a-c)=$ $(c-b)^{T}[2(a-b)+(b-c)]$, the first term on the right-hand side of (B.5) can be transformed as

$$
\begin{aligned}
& \frac{1}{2 q_{1}} \int_{0}^{t}\left[\widetilde{W}_{k}^{T} \widetilde{W}_{k}-\widetilde{W}_{k-1}^{T} \widetilde{W}_{k-1}\right] \mathrm{d} \sigma \\
& =\int_{0}^{t} 2 z_{k}^{T} P B \widetilde{W}_{k}^{T} \phi\left(\widehat{x}_{k}\right) \mathrm{d} \sigma \\
& \quad-2 q_{1} \int_{0}^{t}\left(z_{k}^{T} P B\right)^{2}\left\|\phi\left(\widehat{x}_{k}\right)\right\|^{2} \mathrm{~d} \sigma .
\end{aligned}
$$

Taking mixed type adaptive law (45) into consideration, we can obtain

$$
\begin{aligned}
& \frac{\gamma}{2 q_{2}} \int_{0}^{t}\left[\widetilde{W}_{2, k}^{T} \widetilde{W}_{2, k}-\widetilde{W}_{2, k-1}^{T} \widetilde{W}_{2, k-1}\right] \mathrm{d} \sigma \\
& +\frac{(1-\gamma)}{2 q_{2}}\left[\widetilde{W}_{2, k}^{T} \widetilde{W}_{2, k}-\widetilde{W}_{2, k-1}^{T} \widetilde{W}_{2, k-1}\right] \\
& =\frac{\gamma}{2 q_{2}} \int_{0}^{t}\left[\widetilde{W}_{2, k}^{T} \widetilde{W}_{2, k}-\widetilde{W}_{2, k-1}^{T} \widetilde{W}_{2, k-1}\right] \mathrm{d} \sigma \\
& +\frac{(1-\gamma)}{q_{2}} \int_{0}^{t} \widetilde{W}_{2, k}^{T} \dot{\bar{W}}_{2, k} \mathrm{~d} \sigma \\
& +\frac{(1-\gamma)}{2 q_{2}}\left[\widetilde{W}_{2, k}^{T}(0) \widetilde{W}_{2, k}(0)-\widetilde{W}_{2, k-1}^{T} \widetilde{W}_{2, k-1}\right] \\
& =\int_{0}^{t} b \tanh ^{2}\left(\frac{s_{k}}{\eta}\right) \widetilde{W}_{2, k}^{T} \phi_{2}\left(y_{k}\right) \mathrm{d} \sigma \\
& -\frac{\gamma}{q_{2}} \int_{0}^{t} \widetilde{W}_{2, k}^{T}\left(\widehat{W}_{2, k}-\widehat{W}_{2, k-1}\right) \mathrm{d} \sigma \\
& +\frac{\gamma}{2 q_{2}} \int_{0}^{t}\left[\widetilde{W}_{2, k}^{T} \widetilde{W}_{2, k}-\widetilde{W}_{2, k-1}^{T} \widetilde{W}_{2, k-1}\right] \mathrm{d} \sigma \\
& +\frac{(1-\gamma)}{2 q_{2}}\left[\widetilde{W}_{2, k}^{T}(0) \widetilde{W}_{2, k}(0)-\widetilde{W}_{2, k-1}^{T} \widetilde{W}_{2, k-1}\right] \\
& =\int_{0}^{t} b \tanh ^{2}\left(\frac{s_{k}}{\eta}\right) \widetilde{W}_{2, k}^{T} \phi_{2}\left(y_{k}\right) \mathrm{d} \sigma \\
& -\frac{\gamma}{q_{2}} \int_{0}^{t} \widetilde{W}_{2, k}^{T}\left(\widetilde{W}_{2, k}-\widetilde{W}_{2, k-1}\right) \mathrm{d} \sigma \\
& +\frac{\gamma}{2 q_{2}} \int_{0}^{t}\left[\widetilde{W}_{2, k}^{T} \widetilde{W}_{2, k}-\widetilde{W}_{2, k-1}^{T} \widetilde{W}_{2, k-1}\right] \mathrm{d} \sigma \\
& +\frac{(1-\gamma)}{2 q_{2}}\left[\widetilde{W}_{2, k}^{T}(0) \widetilde{W}_{2, k}(0)-\widetilde{W}_{2, k-1}^{T} \widetilde{W}_{2, k-1}\right] \\
& =\int_{0}^{t} b \tanh ^{2}\left(\frac{s_{k}}{\eta}\right) \widetilde{W}_{2, k}^{T} \phi_{2}\left(y_{k}\right) \mathrm{d} \sigma \\
& -\frac{\gamma}{2 q_{2}} \int_{0}^{t}\left(\widetilde{W}_{2, k}-\widetilde{W}_{2, k-1}\right)^{T}\left(\widetilde{W}_{2, k}-\widetilde{W}_{2, k-1}\right) \mathrm{d} \sigma \\
& +\frac{(1-\gamma)}{2 q_{2}}\left[\widetilde{W}_{2, k}^{T}(0) \widetilde{W}_{2, k}(0)-\widetilde{W}_{2, k-1}^{T} \widetilde{W}_{2, k-1}\right] \\
& \leq \int_{0}^{t} b \tanh ^{2}\left(\frac{s_{k}}{\eta}\right) \widetilde{W}_{2, k}^{T} \phi_{2}\left(y_{k}\right) \mathrm{d} \sigma \\
& +\frac{(1-\gamma)}{2 q_{2}}\left[\widetilde{W}_{2, k}^{T}(0) \widetilde{W}_{2, k}(0)-\widetilde{W}_{2, k-1}^{T} \widetilde{W}_{2, k-1}\right] .
\end{aligned}
$$

Substituting (B.6) and (B.7) back into (B.5) and using (B.3), it follows that

$\Delta E_{k}(t)$

$$
\leq \int_{0}^{t} 2 z_{k}^{T} P B \widetilde{W}_{k}^{T} \phi\left(\widehat{x}_{k}\right) \mathrm{d} \sigma
$$




$$
\begin{aligned}
& +\int_{0}^{t} b \tanh ^{2}\left(\frac{s_{k}}{\eta}\right) \widetilde{W}_{2, k}^{T} \phi_{2}\left(y_{k}\right) \mathrm{d} \sigma \\
& +\frac{(1-\gamma)}{2 q_{2}}\left[\widetilde{W}_{2, k}^{T}(0) \widetilde{W}_{2, k}(0)-\widetilde{W}_{2, k-1}^{T} \widetilde{W}_{2, k-1}\right] \\
\leq & -V_{k}(t)+V_{k}(0)-K \int_{0}^{t} s_{k}^{2} \mathrm{~d} \sigma \\
& -\lambda_{\min }(Q) \int_{0}^{t}\left\|z_{k}\right\|^{2} \mathrm{~d} \sigma \\
& +\frac{(1-\gamma)}{2 q_{2}}\left[\widetilde{W}_{2, k}^{T}(0) \widetilde{W}_{2, k}(0)-\widetilde{W}_{2, k-1}^{T} \widetilde{W}_{2, k-1}\right] \\
\leq & V_{k}(0) \\
& +\frac{(1-\gamma)}{2 q_{2}}\left[\widetilde{W}_{2, k}^{T}(0) \widetilde{W}_{2, k}(0)-\widetilde{W}_{2, k-1}^{T} \widetilde{W}_{2, k-1}\right] .
\end{aligned}
$$

From Assumptions 5, 11, and 12, it is obvious that $V_{k}(0)=$ 0 . Letting $t=T$ in (B.8), according to $\widehat{W}_{2, k}(0)=\widehat{W}_{2, k-1}(T)$, $\widehat{W}_{2,1}(0)=0$, we can have

$$
\begin{aligned}
\Delta E_{k}(T) \leq & -V_{k}(T)-K \int_{0}^{T} s_{k}^{2} \mathrm{~d} \sigma \\
& -\lambda_{\min }(Q) \int_{0}^{T}\left\|z_{k}\right\|^{2} \mathrm{~d} \sigma \\
\leq & -K \int_{0}^{T} s_{k}^{2} \mathrm{~d} \sigma-\lambda_{\min }(Q) \int_{0}^{T}\left\|z_{k}\right\|^{2} \mathrm{~d} \sigma \leq 0 .
\end{aligned}
$$

Inequality (B.9) indicates that $E_{k}(T)$ is decreasing along iteration axis. Thus the boundedness of $E_{k}(T)$ can be ensured provided that $E_{1}(T)$ is finite.

(2) The Finiteness of $E_{1}(T)$. According to (B.4), we know

$$
\begin{aligned}
E_{1}(t)= & \frac{1}{2 q_{1}} \int_{0}^{t} \widetilde{W}_{1}^{T} \widetilde{W}_{1} \mathrm{~d} \sigma+\frac{\gamma}{2 q_{2}} \int_{0}^{t} \widetilde{W}_{2,1}^{T} \widetilde{W}_{2,1} \mathrm{~d} \sigma \\
& +\frac{(1-\gamma)}{2 q_{2}} \widetilde{W}_{2,1}^{T} \widetilde{W}_{2,1} .
\end{aligned}
$$

Taking the time derivative of $E_{1}(t)$ yields

$$
\begin{gathered}
\dot{E}_{1}(t)=\frac{1}{2 q_{1}} \widetilde{W}_{1}^{T} \widetilde{W}_{1}+\frac{\gamma}{2 q_{2}} \widetilde{W}_{2,1}^{T} \widetilde{W}_{2,1} \\
+\frac{(1-\gamma)}{q_{2}} \widetilde{W}_{2,1}^{T} \dot{\widetilde{W}}_{2,1} .
\end{gathered}
$$

Recalling parameter adaptive learning laws (44) and (45), we have $\widehat{W}_{1}(t)=2 q_{1} z_{1}^{T} P B \phi\left(\widehat{x}_{1}\right)$ and $(1-\gamma) \dot{\widehat{W}}_{2,1}=-\gamma \widehat{W}_{2,1}+$ $q_{2} b \tanh ^{2}\left(s_{1} / \eta(t)\right) \phi_{2}\left(y_{1}\right)$; then we obtain

$$
\begin{aligned}
& \frac{1}{2 q_{1}} \widetilde{W}_{1}^{T} \widetilde{W}_{1}=\frac{1}{2 q_{1}}\left[\widetilde{W}_{1}^{T} \widetilde{W}_{1}-2 \widetilde{W}_{1}^{T} \widehat{W}_{1}\right]+\frac{1}{q_{1}} \widetilde{W}_{1}^{T} \widehat{W}_{1} \\
& =\frac{1}{2 q_{1}}\left[\left(\widehat{W}_{1}^{T}-W^{*}\right)^{T}\left(\widehat{W}_{1}^{T}-W^{*}\right)\right. \\
& \left.-2\left(\widehat{W}_{1}^{T}-W^{*}\right)^{T} \widehat{W}_{1}\right]+2 q_{1} z_{1}^{T} P B \widetilde{W}_{1}^{T} \phi\left(\widehat{x}_{1}\right) \\
& =\frac{1}{2 q_{1}}\left[-\widehat{W}_{1}^{T} \widehat{W}_{1}+W^{* T} W^{*}\right] \\
& +2 q_{1} z_{1}^{T} P B \widetilde{W}_{1}^{T} \phi\left(\widehat{x}_{1}\right) \leq \frac{1}{2 q_{1}} W^{* T} W^{*} \\
& +2 q_{1} z_{1}^{T} P B \widetilde{W}_{1}^{T} \phi\left(\widehat{x}_{1}\right) \\
& \frac{\gamma}{2 q_{2}} \widetilde{W}_{2,1}^{T} \widetilde{W}_{2,1}+\frac{(1-\gamma)}{q_{2}} \widetilde{W}_{2,1}^{T} \dot{\bar{W}}_{2,1}=\frac{\gamma}{2 q_{2}} \widetilde{W}_{2,1}^{T} \widetilde{W}_{2,1} \\
& -\frac{\gamma}{q_{2}} \widetilde{W}_{2,1}^{T} \widehat{W}_{2,1}+b \tanh ^{2}\left(\frac{s_{1}}{\eta(t)}\right) \widetilde{W}_{2,1}^{T} \phi_{2}\left(y_{1}\right) \\
& =\frac{\gamma}{2 q_{2}}\left[\widetilde{W}_{2,1}^{T} \widetilde{W}_{2,1}-2 \widetilde{W}_{2,1}^{T} \widehat{W}_{2,1}+\widehat{W}_{2,1}^{T} \widehat{W}_{2,1}\right]-\frac{\gamma}{2 q_{2}} \\
& \cdot \widehat{W}_{2,1}^{T} \widehat{W}_{2,1}+b \tanh ^{2}\left(\frac{s_{1}}{\eta(t)}\right) \widetilde{W}_{2,1}^{T} \phi_{2}\left(y_{1}\right) \\
& \leq \frac{\gamma}{2 q_{2}}\left[\widehat{W}_{2,1}-\widetilde{W}_{2,1}\right]^{T}\left[\widehat{W}_{2,1}-\widetilde{W}_{2,1}\right] \\
& +b \tanh ^{2}\left(\frac{s_{1}}{\eta(t)}\right) \widetilde{W}_{2,1}^{T} \phi_{2}\left(y_{1}\right)=\frac{\gamma}{2 q_{2}} W_{2}^{* T} W_{2}^{*} \\
& +b \tanh ^{2}\left(\frac{s_{1}}{\eta(t)}\right) \widetilde{W}_{2,1}^{T} \phi_{2}\left(y_{1}\right) \text {. }
\end{aligned}
$$

Combining (B.12) and (B.13), if follows from (B.11) that

$$
\begin{aligned}
\dot{E}_{1}(t) \leq & \frac{1}{2 q_{1}} W^{* T} W^{*}+2 q_{1} z_{1}^{T} P B \widetilde{W}_{1}^{T} \phi\left(\widehat{x}_{1}\right) \\
& +\frac{\gamma}{2 q_{2}} W_{2}^{* T} W_{2}^{*} \\
& +b \tanh ^{2}\left(\frac{s_{1}}{\eta(t)}\right) \widetilde{W}_{2,1}^{T} \phi_{2}\left(y_{1}\right) \\
\leq & -\dot{V}_{1}-\lambda_{\min }(Q)\left\|z_{1}\right\|^{2}-K s_{1}^{2}+\frac{1}{2 q_{1}} W^{* T} W^{*} \\
& +\frac{\gamma}{2 q_{2}} W_{2}^{* T} W_{2}^{*} .
\end{aligned}
$$


Denote $c_{\max }=\max _{t \in[0, T]}\left\{\left(1 / 2 q_{1}\right) W^{* T}(t) W^{*}(t)+(\gamma /\right.$ $\left.\left.2 q_{2}\right) W_{2}^{* T} W_{2}^{*}\right\}$. Integrating (B.14) over $[0, t]$ leads to

$$
\begin{aligned}
E_{1}(t)-E_{1}(0) \leq & -V_{1}(t)+V_{1}(0) \\
& -\lambda_{\min }(Q) \int_{0}^{t}\left\|z_{1}\right\|^{2} \mathrm{~d} \sigma \\
& -\int_{0}^{t} K s_{1}^{2}(\sigma) \mathrm{d} \sigma+t \cdot c_{\max } .
\end{aligned}
$$

According to $\widehat{W}_{2,1}(0)=0$, we get

$$
E_{1}(0)=\frac{(1-\gamma)}{2 q_{2}} \widetilde{W}_{2,1}^{T}(0) \widetilde{W}_{2,1}(0)=\frac{(1-\gamma)}{2 q_{2}}\left\|W_{2}^{*}\right\|^{2} \text {. }
$$

Substituting (B.16) back into (B.15) results in

$$
\begin{aligned}
E_{1}(t) & \leq t \cdot c_{\max }+\frac{(1-\gamma)}{2 q_{2}}\left\|W_{2}^{*}\right\|^{2} \\
& <T \cdot c_{\max }+\frac{(1-\gamma)}{2 q_{2}}\left\|W_{2}^{*}\right\|^{2}, \quad t \in[0, T]
\end{aligned}
$$

which implies the boundedness of $E_{1}(t)$ on $[0, T]$. Letting $t=$ $T$, we can obtain the boundedness of $E_{1}(T)$ :

$$
E_{1}(T) \leq T \cdot c_{\max }+\frac{(1-\gamma)}{2 q_{2}}\left\|W_{2}^{*}\right\|^{2}<\infty .
$$

Thus, recalling Part (1) we obtain the finiteness of $E_{k}(T)$.

(3) The Finiteness of $E_{k}(t)$. Next we will prove the boundedness of the signals by induction. Firstly, we separate $E_{k}(t)$ into two parts

$$
\begin{aligned}
& E_{k}^{1}(t)=\frac{1}{2 q_{1}} \int_{0}^{t} \widetilde{W}_{k}^{T} \widetilde{W}_{k} \mathrm{~d} \sigma+\frac{\gamma}{2 q_{2}} \int_{0}^{t} \widetilde{W}_{2, k}^{T} \widetilde{W}_{2, k} \mathrm{~d} \sigma, \\
& E_{k}^{2}(t)=\frac{(1-\gamma)}{2 q_{2}} \widetilde{W}_{2, k}^{T} \widetilde{W}_{2, k} .
\end{aligned}
$$

The boundedness of $E_{k}^{1}(T)$ and $E_{k}^{2}(T)$ is guaranteed for all iterations. Consequently, for all $k \in N$, there exist two constants $M_{1}$ and $M_{2}$ satisfying

$$
\begin{aligned}
& E_{k}^{1}(t) \leq E_{k}^{1}(T) \leq M_{1}<\infty, \\
& E_{k}^{2}(T) \leq M_{2} .
\end{aligned}
$$

Then, we have

$$
E_{k}(t)=E_{k}^{1}(t)+E_{k}^{2}(t) \leq M_{1}+E_{k}^{2}(t) .
$$
obtain

On the other hand, from (45) and $E_{k+1}^{2}(0)=E_{k}^{2}(T)$, we

$$
\begin{aligned}
& \Delta E_{k+1}(t) \\
& \quad<\frac{(1-\gamma)}{2 q_{2}}\left[\widetilde{W}_{2, k+1}^{T}(0) \widetilde{W}_{2, k+1}(0)-\widetilde{W}_{2, k}^{T} \widetilde{W}_{2, k}\right] \\
& \quad \leq M_{2}-E_{k}^{2}(t) .
\end{aligned}
$$

Combining (B.21) and (B.22) results in

$$
E_{k+1}(t)=E_{k}(t)+\Delta E_{k+1}(t) \leq M_{1}+M_{2}
$$

As we have proven that $E_{1}(t)$ is bounded, therefore $E_{k}(t)$ is finite. Furthermore, we can obtain the boundedness of $\widehat{W}_{k}(t)$ and $\widehat{W}_{2, k}$.

(4) Learning Convergence Property. Applying (B.9) repeatedly, we have

$$
\begin{aligned}
E_{k}(T)= & E_{1}(T)+\sum_{j=2}^{k} \Delta E_{j}(T) \\
\leq & E_{1}(T)-K \sum_{j=2}^{k} \int_{0}^{T} s_{j}^{2} \mathrm{~d} \sigma \\
& -\lambda_{\min }(Q) \sum_{j=2}^{k} \int_{0}^{T}\left\|z_{j}\right\|^{2} \mathrm{~d} \sigma .
\end{aligned}
$$

We rewrite inequality (B.24) as

$$
\begin{gathered}
\sum_{j=2}^{k} \int_{0}^{T} s_{j}^{2} \mathrm{~d} \sigma \leq \frac{\left(E_{1}(T)-E_{k}(T)\right)}{K} \leq \frac{E_{1}(T)}{K}, \\
\sum_{j=2}^{k} \int_{0}^{T}\left\|z_{j}\right\|^{2} \mathrm{~d} \sigma \leq \frac{\left(E_{1}(T)-E_{k}(T)\right)}{\lambda_{\min }(Q)} \leq \frac{E_{1}(T)}{\lambda_{\min }(Q)} .
\end{gathered}
$$

Taking the limitation of (B.25), it follows that

$$
\begin{aligned}
& \lim _{k \rightarrow \infty} \sum_{j=2}^{k} \int_{0}^{T} s_{j}^{2}(\sigma) \mathrm{d} \sigma \leq \frac{E_{1}(T)}{K}, \\
& \lim _{k \rightarrow \infty} \sum_{j=2}^{k} \int_{0}^{T}\left\|z_{j}\right\|^{2} \mathrm{~d} \sigma \leq \frac{E_{1}(T)}{\lambda_{\min }(Q)} .
\end{aligned}
$$

Since $E_{1}(T)$ is bounded, according to the convergence theorem of the sum of series, $\lim _{k \rightarrow \infty} \int_{0}^{T} s_{k}^{2}(\sigma) \mathrm{d} \sigma=0$ and $\lim _{k \rightarrow \infty} \int_{0}^{T}\left\|z_{k}\right\|^{2} \mathrm{~d} \sigma=0$. It is obvious that $\lim _{k \rightarrow \infty} \int_{0}^{T}\left(y_{k}-\widehat{y}_{k}\right)^{2} \mathrm{~d} \sigma \leq \lim _{k \rightarrow \infty} \int_{0}^{T}\left\|z_{k}\right\|^{2} \mathrm{~d} \sigma=0$, for all $t \in[0, T]$. Besides, $\lim _{k \rightarrow \infty} \int_{0}^{T} s_{k}^{2}(\sigma) \mathrm{d} \sigma=0$ is equivalent to $\lim _{k \rightarrow \infty} \int_{0}^{T}\left|s_{k}(\sigma)\right| \mathrm{d} \sigma=0$ which means that $\lim _{k \rightarrow \infty} \int_{0}^{T}\left|e_{s k}(\sigma)\right| \mathrm{d} \sigma \leq \int_{0}^{T} \eta(\sigma) \mathrm{d} \sigma$ and $\lim _{k \rightarrow \infty} \int_{0}^{T}\left(e_{s k}(\sigma)\right)^{2} \mathrm{~d} \sigma \leq \int_{0}^{T} \eta^{2}(\sigma) \mathrm{d} \sigma$. From $\int_{0}^{t} s_{k}^{2}(\sigma) \mathrm{d} \sigma \leq$ $\int_{0}^{T} s_{k}^{2}(\sigma) \mathrm{d} \sigma$ and $\int_{0}^{t}\left\|z_{k}(\sigma)\right\|^{2} \mathrm{~d} \sigma \leq \int_{0}^{T}\left\|z_{k}(\sigma)\right\|^{2} \mathrm{~d} \sigma$, we can get the boundedness of $s_{k}(t)$ and $\left\|z_{k}\right\|$ in $L_{T}^{2}$-norm, which further implies that $x_{k}(t)$ and $\widehat{x}_{k}(t)$ are bounded. Based on the above reasoning, we can arrive at the fact that $v_{k}(t)$ is bounded.

Synthesizing the discussions in two cases, we can draw the conclusion that, for two cases, the proposed control algorithm is able to guarantee that all closed-loop signals are bounded, and $\lim _{k \rightarrow \infty} \int_{0}^{T}\left\|z_{k}\right\|^{2} \mathrm{~d} \sigma=0$ and $\lim _{k \rightarrow \infty} \int_{0}^{T}\left(e_{s k}(\sigma)\right)^{2} \mathrm{~d} \sigma \leq$ 
$\varepsilon_{e}, \varepsilon_{e}=\int_{0}^{T}\left((1+m) \eta_{1}(\sigma)\right)^{2} \mathrm{~d} \sigma=(1 / 2 K)(1+m)^{2} \varepsilon^{2}\left(1-e^{-2 K T}\right) \leq$ $(1 / 2 K)(1+m)^{2} \varepsilon^{2}=\varepsilon_{e s k}$. Furthermore, the bound of $e_{s \infty}(t)$ will satisfy $\lim _{k \rightarrow \infty}\left|e_{s k}(t)\right|=e_{s \infty}(t)=(1+m) \varepsilon e^{-K t}$, for all $t \in[0, T]$.

(5) Transient Performance. Next, we determine an upper bound of the error vector $e_{k}(t)$. Define the vector $\zeta_{k}(t)=$ $\left[e_{1, k}(t), e_{2, k}(t), \ldots, e_{n-1, k}(t)\right]^{T}$; then a state representation of $e_{s k}(t)=\left[\begin{array}{ll}\Lambda^{T} & 1\end{array}\right] e_{k}(t)$ can be expressed as

$$
\dot{\zeta}_{k}(t)=A_{s} \zeta_{k}(t)+b_{s} e_{s k}(t)+K_{s} z_{1, k}
$$

where

$$
\begin{aligned}
& A_{s}=\left[\begin{array}{rrrr}
0 & 1 & \cdots & 0 \\
\vdots & \vdots & \ddots & \vdots \\
0 & 0 & \cdots & 1 \\
-\lambda_{1} & -\lambda_{2} & \cdots & -\lambda_{n-1}
\end{array}\right] \in R^{(n-1) \times(n-1)} \\
& b_{s}=\left[\begin{array}{c}
0 \\
\vdots \\
0 \\
1
\end{array}\right] \in R^{n-1}, \\
& K_{s}=\left[\begin{array}{c}
k_{1} \\
\vdots \\
k_{n-1}
\end{array}\right]
\end{aligned}
$$

with $A_{s}$ as a stable matrix. In addition, there are two constants $k_{0}>0$ and $\lambda_{0}>0$ such that $\left\|e^{A_{s} t}\right\| \leq k_{0} e^{-\lambda_{0} t}$ [48]. The solution for $\dot{\zeta}_{k}(t)$ is

$$
\begin{aligned}
\zeta_{k}(t)= & e^{A_{s} t} \zeta_{k}(0)+\int_{0}^{t} e^{A_{s}(t-\sigma)} b_{s} e_{s k}(\sigma) \mathrm{d} \sigma \\
& +\int_{0}^{t} e^{A_{s}(t-\sigma)} K_{s} z_{1, k}(\sigma) \mathrm{d} \sigma .
\end{aligned}
$$

Consequently, it follows that

$$
\begin{aligned}
\left\|\zeta_{k}(t)\right\| \leq & k_{0}\left\|\zeta_{k}(0)\right\| e^{-\lambda_{0} t} \\
& +k_{0} \int_{0}^{t} e^{-\lambda_{0}(t-\sigma)}\left|e_{s k}(\sigma)\right| \mathrm{d} \sigma \\
& +k_{0}\left\|K_{s}\right\| \int_{0}^{t} e^{-\lambda_{0}(t-\sigma)}\left|z_{1, k}(\sigma)\right| \mathrm{d} \sigma .
\end{aligned}
$$

When we choose suitable parameters such that $\lambda_{0}>K$, from $\lim _{k \rightarrow \infty}\left|e_{s k}(t)\right| \leq(1+m) \eta(t)$, we can have

$$
\begin{aligned}
\left\|\zeta_{\infty}(t)\right\|= & k_{0}\left\|\zeta_{\infty}(0)\right\| e^{-\lambda_{0} t} \\
& +k_{0} \int_{0}^{t} e^{-\lambda_{0}(t-\sigma)}\left|e_{s \infty}(\sigma)\right| \mathrm{d} \sigma \\
& +k_{0}\left\|K_{s}\right\| \int_{0}^{t} e^{-\lambda_{0}(t-\sigma)}\left|z_{1, \infty}(\sigma)\right| \mathrm{d} \sigma \\
\leq & k_{0}\left\|\zeta_{\infty}(0)\right\| \\
& +(1+m) \varepsilon k_{0} \int_{0}^{t} e^{-\lambda_{0}(t-\sigma)} e^{-K \sigma} \mathrm{d} \sigma+0 \\
= & k_{0}\left\|\zeta_{\infty}(0)\right\| \\
& +(1+m) \varepsilon k_{0} \frac{1}{\lambda_{0}-K}\left(e^{-K t}-e^{-\lambda_{0} t}\right) \\
\leq & k_{0}\left\|\zeta_{\infty}(0)\right\|+\frac{1}{\lambda_{0}-K}(1+m) \varepsilon k_{0} .
\end{aligned}
$$

Noting $e_{s k}(t)=\left[\begin{array}{ll}\Lambda^{T} & 1\end{array}\right] e_{k}(t)$ and $e_{k}(t)=\left[\begin{array}{ll}\zeta_{k}^{T}(t) & e_{n, k}(t)\end{array}\right]^{T}$, we have

$$
\begin{aligned}
\left\|e_{k}(t)\right\| & \leq\left\|\zeta_{k}(t)\right\|+\left|e_{n, k}(t)\right| \\
& =\left\|\zeta_{k}(t)\right\|+\left|e_{s k}(t)-\Lambda^{T} \zeta_{k}(t)\right| \\
& \leq(1+\|\Lambda\|)\left\|\zeta_{k}(t)\right\|+\left|e_{s k}(t)\right|
\end{aligned}
$$

Considering the two inequalities above, we can obtain

$$
\begin{aligned}
&\left\|e_{\infty}(t)\right\| \leq(1+\|\Lambda\|)\left\|\zeta_{\infty}(t)\right\|+\left|e_{s \infty}(t)\right| \\
& \leq(1+\|\Lambda\|)\left(k_{0}\left\|\zeta_{\infty}(0)\right\|+\frac{1}{\lambda_{0}-K}(1+m) \varepsilon k_{0}\right) \\
&+(1+m) \eta(t) \\
& \leq(1+\|\Lambda\|)\left(k_{0} \sum_{i=1}^{n-1} \sqrt{\varepsilon_{i}^{2}}+\frac{1}{\lambda_{0}-K}(1+m) \varepsilon k_{0}\right) \\
&+(1+m) \eta(t) .
\end{aligned}
$$

Since $\zeta_{k}(t)=\left[e_{1, k}(t), e_{2, k}(t), \ldots, e_{n-1, k}(t)\right]^{T}$, then it follows from (B.28) that

$$
\begin{aligned}
\left|e_{1, k}(t)\right| \leq & \left\|\zeta_{k}(t)\right\| \\
\leq & k_{0}\left\|\zeta_{k}(0)\right\| e^{-\lambda_{0} t} \\
& +k_{0} \int_{0}^{t} e^{-\lambda_{0}(t-\sigma)}\left|e_{s k}(\sigma)\right| \mathrm{d} \sigma .
\end{aligned}
$$


When $k \rightarrow \infty$, it can be shown that

$$
\begin{aligned}
\left|e_{1, \infty}(t)\right| \leq & \left\|\zeta_{\infty}(t)\right\| \\
\leq & k_{0}\left\|\zeta_{\infty}(0)\right\| e^{-\lambda_{0} t} \\
& +k_{0} \int_{0}^{t} e^{-\lambda_{0}(t-\sigma)}\left|e_{s \infty}(\sigma)\right| \mathrm{d} \sigma \\
\leq & k_{0}\left\|\zeta_{\infty}(0)\right\| e^{-\lambda_{0} t} \\
& +(1+m) \varepsilon k_{0} \int_{0}^{t} e^{-\lambda_{0}(t-\sigma)} e^{-K \sigma} \mathrm{d} \sigma \\
= & k_{0}\left\|\zeta_{\infty}(0)\right\| e^{-\lambda_{0} t} \\
& +(1+m) \varepsilon k_{0} \frac{1}{\lambda_{0}-K}\left(e^{-K t}-e^{-\lambda_{0} t}\right) \\
\leq & k_{0} \sum_{i=1}^{n-1} \sqrt{\varepsilon_{i}^{2}} e^{-\lambda_{0} t} \\
& +(1+m) \varepsilon k_{0} \frac{1}{\lambda_{0}-K}\left(e^{-K t}-e^{-\lambda_{0} t}\right) .
\end{aligned}
$$

Define $\chi_{k}=\left[\chi_{1, k}, \ldots, \chi_{n, k}\right]^{T}=x_{k}-x_{d}$; then we can know that $\chi_{k}=z_{k}+e_{k}$. From $\lim _{k \rightarrow \infty} \int_{0}^{T}\left\|z_{k}\right\|^{2} \mathrm{~d} \sigma=0$ we can have $\lim _{k \rightarrow \infty}\left\|z_{k}(t)\right\|=0$; that is, $\left\|z_{\infty}(t)\right\|=0, t \in[0, T]$. Consequently, it is obvious that

$$
\begin{aligned}
\left\|\chi_{\infty}\right\| & \leq\left\|z_{\infty}\right\|+\left\|e_{\infty}\right\| \\
\leq & (1+\|\Lambda\|)\left(k_{0} \sum_{i=1}^{n-1} \sqrt{\varepsilon_{i}^{2}}+\frac{1}{\lambda_{0}-K}(1+m) \varepsilon k_{0}\right) \\
& +(1+m) \eta(t), \\
\left|\chi_{1, \infty}\right| & =\left|y_{\infty}-y_{d}\right| \leq\left|z_{1, \infty}\right|+\left|e_{1, \infty}\right| \\
\leq & k_{0} \sum_{i=1}^{n-1} \sqrt{\varepsilon_{i}^{2}} e^{-\lambda_{0} t} \\
& +(1+m) \varepsilon k_{0} \frac{1}{\lambda_{0}-K}\left(e^{-K t}-e^{-\lambda_{0} t}\right) .
\end{aligned}
$$

This concludes the proof.

\section{Conflict of Interests}

The authors declare that there is no conflict of interests regarding the publication of this paper.

\section{References}

[1] H.-S. Lee and Z. Bien, "A note on convergence property of iterative learning controller with respect to sup norm," Automatica, vol. 33, no. 8, pp. 1591-1593, 1997.

[2] Y. Chen, Z. Gong, and C. Wen, "Analysis of a high-order iterative learning control algorithm for uncertain nonlinear systems with state delays," Automatica, vol. 34, no. 3, pp. 345353, 1998 .
[3] M. Sun and D. Wang, "Iterative learning control with initial rectifying action," Automatica, vol. 38, no. 8, pp. 1177-1182, 2002.

[4] C.-J. Chien and J.-S. Liu, "A P-type iterative learning controller for robust output tracking of nonlinear time-varying systems," International Journal of Control, vol. 64, no. 2, pp. 319-334, 1996.

[5] J.-X. Xu and Y. Tan, "A composite energy function-based learning control approach for nonlinear systems with timevarying parametric uncertainties," IEEE Transactions on Automatic Control, vol. 47, no. 11, pp. 1940-1945, 2002.

[6] A. Tayebi, "Adaptive iterative learning control for robot manipulators," Automatica, vol. 40, no. 7, pp. 1195-1203, 2004.

[7] J.-X. Xu, Y. Tan, and T.-H. Lee, "Iterative learning control design based on composite energy function with input saturation," Automatica, vol. 40, no. 8, pp. 1371-1377, 2004.

[8] R. H. Chi, Z. S. Hou, and J. X. Xu, "Adaptive ILC for a class of discrete-time systems with iteration-varying trajectory and random initial condition," Automatica, vol. 44, no. 8, pp. 22072213,2008

[9] C.-J. Chien, "A combined adaptive law for fuzzy iterative learning control of nonlinear systems with varying control tasks," IEEE Transactions on Fuzzy Systems, vol. 16, no. 1, pp. 40$51,2008$.

[10] Y.-C. Wang and C.-J. Chien, "Decentralized adaptive fuzzy neural iterative learning control for nonaffine nonlinear interconnected systems," Asian Journal of Control, vol. 13, no. 1, pp. 94-106, 2011.

[11] S. S. Ge, F. Hong, and T. H. Lee, "Robust adaptive control of nonlinear systems with unknown time delays," Automatica, vol. 41, no. 7, pp. 1181-1190, 2005.

[12] T. P. Zhang and S. S. Ge, "Adaptive neural control of MIMO nonlinear state time-varying delay systems with unknown dead-zones and gain signs," Automatica, vol. 43, no. 6, pp. 10211033, 2007.

[13] C. C. Hua, G. Feng, and X. P. Guan, "Robust controller design of a class of nonlinear time delay systems via backstepping method," Automatica, vol. 44, no. 2, pp. 567-573, 2008.

[14] X. D. Ye, "Adaptive stabilization of time-delay feedforward nonlinear systems," Automatica, vol. 47, no. 5, pp. 950-955, 2011.

[15] J. Na, "Adaptive prescribed performance control of nonlinear systems with unknown dead zone," International Journal of Adaptive Control and Signal Processing, vol. 27, no. 5, pp. 426446, 2013.

[16] Z.-Y. Sun and Y.-G. Liu, "Adaptive control design for a class of uncertain high-order nonlinear systems with time delay," Asian Journal of Control, vol. 17, no. 2, pp. 535-543, 2015.

[17] W. S. Chen and L. Zhang, "Adaptive Iterative learning control for nonlinearly parameterized systems with unknown timevarying delays," International Journal of Control, Automation and Systems, vol. 8, no. 2, pp. 177-186, 2010.

[18] J.-M. Wei, Y.-A. Hu, and M.-M. Sun, "A new AILC for a class of nonlinearly parameterized systems with unknown delays and input dead-zone," Journal of Applied Mathematics, vol. 2014, Article ID 238018, 14 pages, 2014.

[19] J. M. Wei, Y. A. Hu, and M. M. Sun, "Adaptive iterative learning control for a class of nonlinear time-varying systems with unknown delays and input dead-zone," IEEE/CAA Journal of Automatica Sinica, vol. 1, no. 3, pp. 302-314, 2014.

[20] C. Wen, J. Zhou, Z. Liu, and H. Su, "Robust adaptive control of uncertain nonlinear systems in the presence of input saturation and external disturbance," IEEE Transactions on Automatic Control, vol. 56, no. 7, pp. 1672-1678, 2011. 
[21] M. Chen, S. S. Ge, and B. V. E. How, "Robust adaptive neural network control for a class of uncertain MIMO nonlinear systems with input nonlinearities," IEEE Transactions on Neural Networks, vol. 21, no. 5, pp. 796-812, 2010.

[22] M. Chen, S. S. Ge, and B. B. Ren, "Adaptive tracking control of uncertain MIMO nonlinear systems with input constraints," Automatica, vol. 47, no. 3, pp. 452-465, 2011.

[23] B. Zhou, H. Gao, Z. Lin, and G.-R. Duan, "Stabilization of linear systems with distributed input delay and input saturation," Automatica, vol. 48, no. 5, pp. 712-724, 2012.

[24] R. Y. Yuan, X. M. Tan, G. L. Fan, and J. Q. Yi, "Robust adaptive neural network control for a class of uncertain nonlinear systems with actuator amplitude and rate saturations," Neurocomputing, vol. 125, pp. 72-80, 2014.

[25] H. Wang, B. Chen, X. Liu, K. Liu, and C. Lin, "Adaptive neural tracking control for stochastic nonlinear strict-feedback systems with unknown input saturation," Information Sciences, vol. 269, pp. 300-315, 2014.

[26] Y. Yang, D. Yue, and Y. S. Xue, "Decentralized adaptive neural output feedback control of a class of large-scale time-delay systems with input saturation," Journal of the Franklin Institute, vol. 352, no. 5, pp. 2129-2151, 2015.

[27] H. H. Ji, Z. H. Hou, and R. K. Zhang, "Adaptive iterative learning control for high-speed trains with unknown speed delays and input saturations," IEEE Transactions on Automation Science and Engineering, 2015.

[28] Y. H. Kim and F. L. Lewis, High-Level Feedback Control with Neural Networks, World Scientific Publishing, River Edge, NJ, USA, 1998.

[29] Y.-J. Liu and N. Zhou, "Observer-based adaptive fuzzy-neural control for a class of uncertain nonlinear systems with unknown dead-zone input," ISA Transactions, vol. 49, no. 4, pp. 462-469, 2010.

[30] S. C. Tong, Y. Li, Y. M. Li, and Y. J. Liu, "Observer-based adaptive fuzzy backstepping control for a class of stochastic nonlinear strict-feedback systems," IEEE Transactions on Systems, Man, and Cybernetics, Part B: Cybernetics, vol. 41, no. 6, pp. 1693-1704, 2011.

[31] B. Zhou, Z.-Y. Li, and Z. L. Lin, "Observer based output feedback control of linear systems with input and output delays," Automatica, vol. 49, no. 7, pp. 2039-2052, 2013.

[32] A. Boulkroune, M. Tadjine, M. M'Saad, and M. Farza, "Design of a unified adaptive fuzzy observer for uncertain nonlinear systems," Information Sciences, vol. 265, pp. 139-153, 2014.

[33] C. C. Hua, C. X. Yu, and X. P. Guan, "Neural network observerbased networked control for a class of nonlinear systems," Neurocomputing, vol. 133, pp. 103-110, 2014.

[34] W. X. Shi, "Observer-based fuzzy adaptive control for multiinput multi-output nonlinear systems with a nonsymmetric control gain matrix and unknown control direction," Fuzzy Sets and Systems, vol. 263, pp. 1-26, 2015.

[35] B. Chen, C. Lin, X. P. Liu, and K. F. Liu, "Observer-based adaptive fuzzy control for a class of nonlinear delayed systems," IEEE Transactions on Systems, Man, and Cybernetics: Systems, 2015.

[36] A. Tayebi and J.-X. Xu, "Observer-based iterative learning control for a class of time-varying nonlinear systems," IEEE Transactions on Circuits and Systems. I: Fundamental Theory and Applications, vol. 50, no. 3, pp. 452-455, 2003.

[37] J.-X. Xu and J. Xu, "Observer based learning control for a class of nonlinear systems with time-varying parametric uncertainties,"
IEEE Transactions on Automatic Control, vol. 49, no. 2, pp. 275281, 2004.

[38] Y.-C. Wang and C.-J. Chien, "An observer based adaptive iterative learning control for robotic systems," in Proceedings of the IEEE International Conference on Fuzzy Systems (FUZZ '11), pp. 2876-2881, Taipei, Taiwan, June 2011.

[39] C.-J. Chien and Y.-C. Wang, "An observer-based fuzzy neural network adaptive ILC for nonlinear systems," in Proceedings of the 13th International Conference on Control, Automation and Systems (ICCAS '13), pp. 226-232, Kimdaejung Convention Center, Gwangju, Republic of Korea, October 2013.

[40] Y. C. Wang, C. J. Chien, and M. J. Er, "An observer-based adaptive iterative learning controller for MIMO nonlinear systems with delayed output," in Proceedings of the 2014 13th International Conference on Control, Automation, Robotics \& Vision, pp. 157-162, Marina BaySands, Singapore, December 2014.

[41] Y. C. Wang and C. J. Chien, "An observer-based model reference adaptive iterative learning controller for MIMO nonlinear systems," in Proceedings of the 11th IEEE International Conference on Control \& Automation (ICCA '14), pp. 1168-1173, Taichung, Taiwan, June 2014.

[42] W.-S. Chen, R.-H. Li, and J. Li, "Observer-based adaptive iterative learning control for nonlinear systems with time-varying delays," International Journal of Automation and Computing, vol. 7, no. 4, pp. 438-446, 2010.

[43] M. M. Gupta and D. H. Rao, Neuro-Control Systems: Theory and Applications, IEEE Press, New York, NY, USA, 1994.

[44] M. X. Sun, "Iterative learning neurocomputing," in Proceedings of the International Conference on Wireless Networks and Information Systems (WNIS '09), pp. 158-161, Shanghai, China, December 2009.

[45] M.-X. Sun, "Time-varying neurocomputing: an iterative learning perspective," in Intelligent Computing Theories and Applications: 8th International Conference, ICIC 2012, Huangshan, China, July 25-29, 2012. Proceedings, vol. 7390 of Lecture Notes in Computer Science, pp. 18-26, Springer, Berlin, Germany, 2012.

[46] S. Boyd, L. E. Ghaoui, E. Feron, and V. Balakrishnan, Linear Matrix Inequalities in System and Control Theory, SIAM, Philadelphia, Pa, USA, 1994.

[47] S. S. Ge and K. P. Tee, "Approximation-based control of nonlinear MIMO time-delay systems," Automatica, vol. 43, no. 1, pp. 31-43, 2007.

[48] S. S. Ge, C. C. Hang, T. H. Lee, and T. Zhang, Stable Adaptive Neural Network Control, Kluwer Academic Publishers, Norwell, Mass, USA, 2001. 


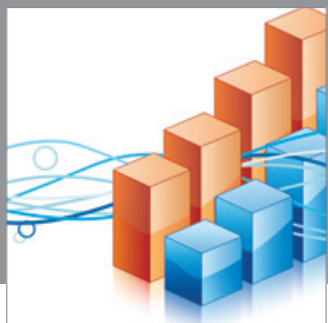

Advances in

Operations Research

mansans

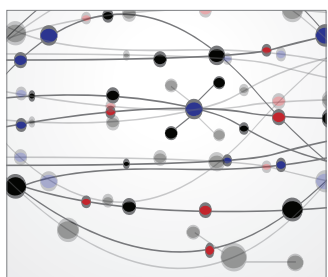

The Scientific World Journal
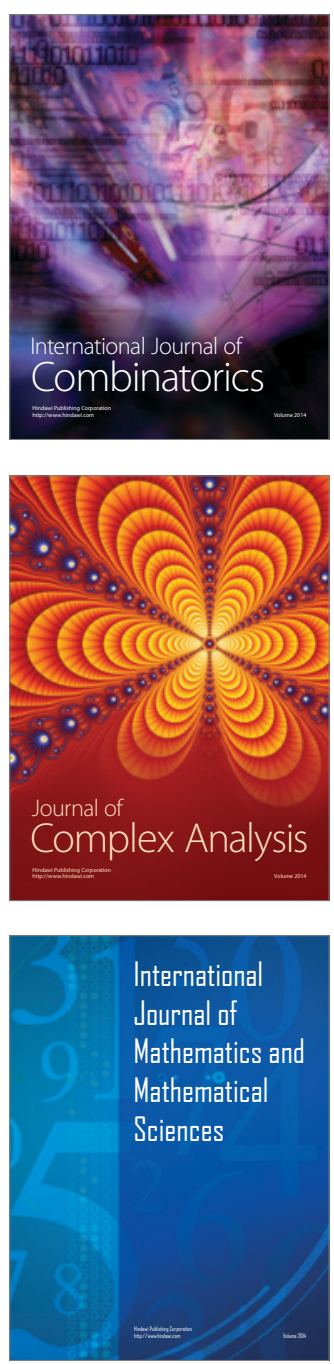
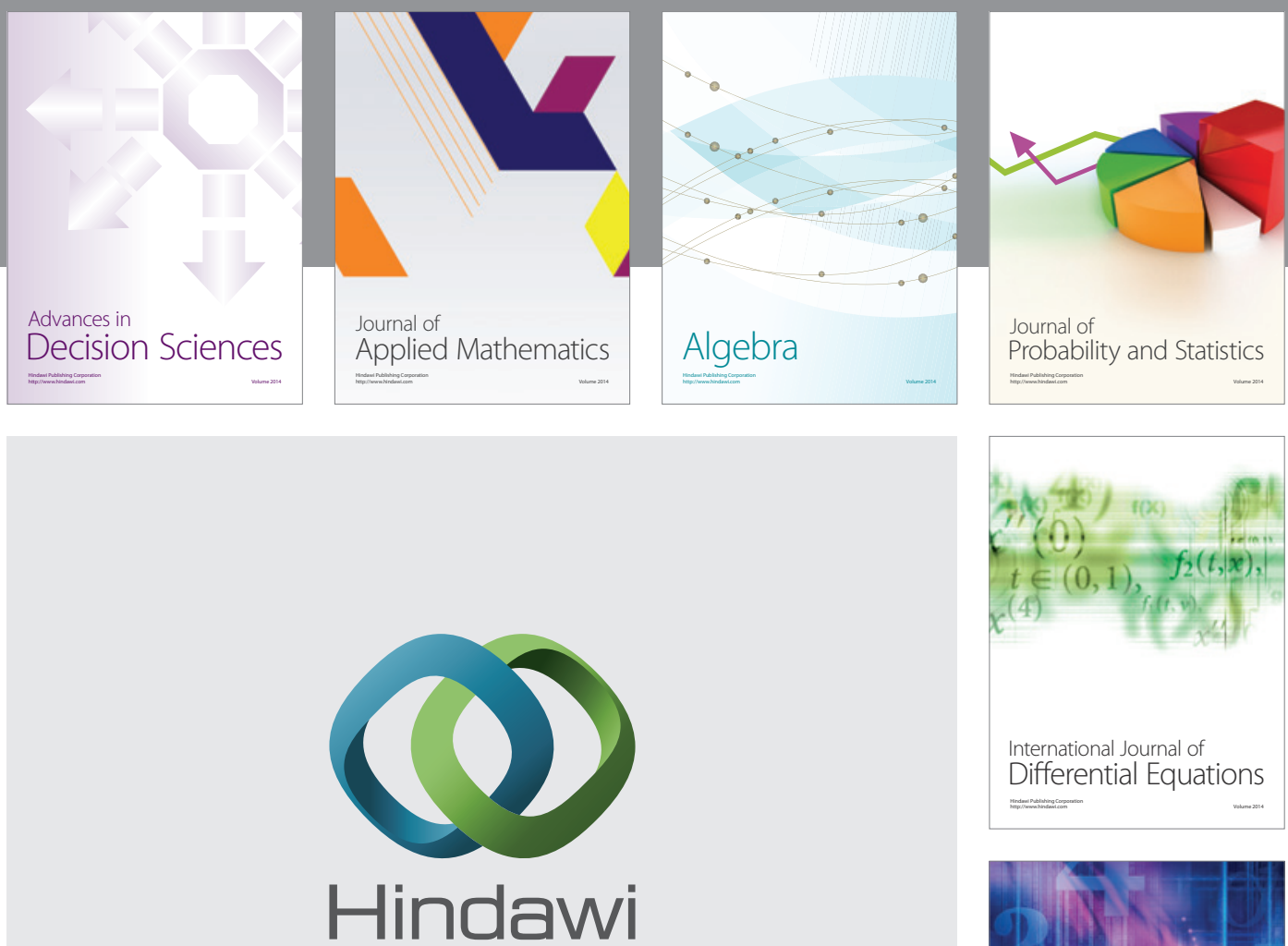

Submit your manuscripts at http://www.hindawi.com
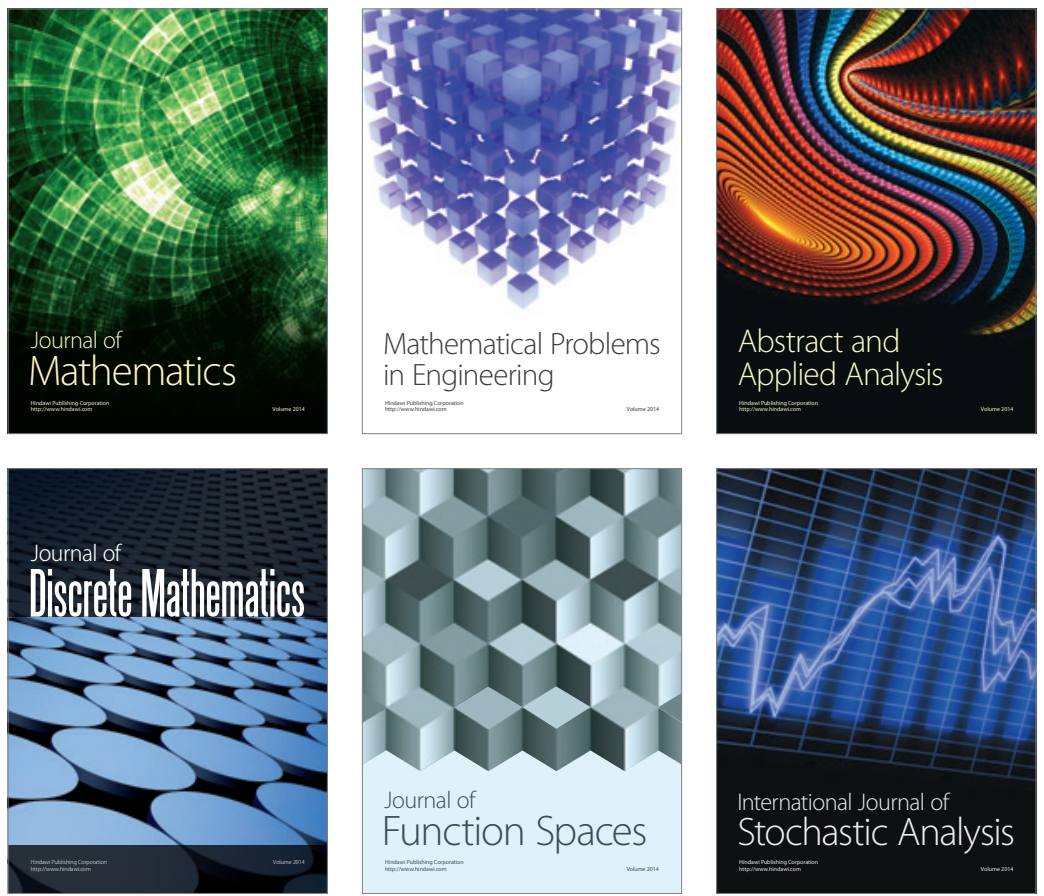

Journal of

Function Spaces

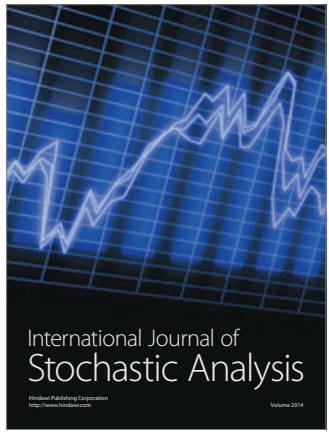

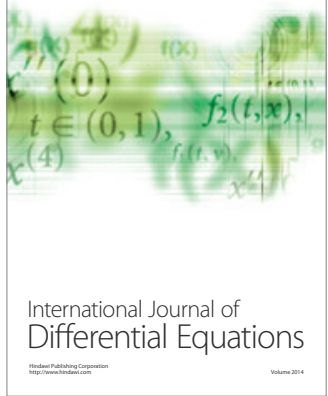
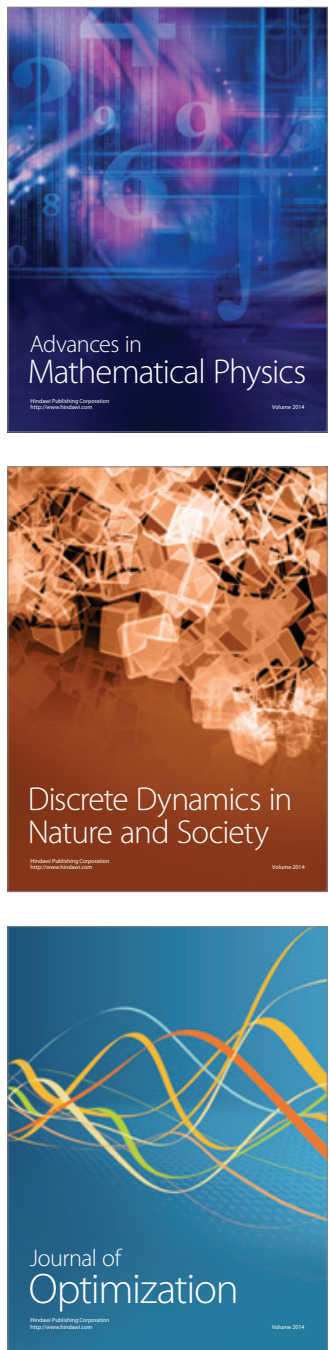